\title{
Discovery of a pulsar-powered bow shock nebula in the Small Magellanic Cloud supernova remnant DEM S5
}

\author{
Rami Z. E. Alsaberi ${ }^{\oplus},{ }^{\star}$ C. Maitra ${ }^{\oplus},{ }^{2}$ M. D. Filipović ${ }^{\oplus},{ }^{1 \star}$ L. M. Bozzetto, ${ }^{1}$ \\ F. Haberl ${ }^{\oplus},{ }^{2}$ P. Maggi, ${ }^{3}$ M. Sasaki ${ }^{\oplus},{ }^{4}$ P. Manjolović, ${ }^{1,5}$ V. Velović, ${ }^{6}$ P. Kavanagh, ${ }^{7}$ N. \\ I. Maxted ${ }^{\odot}, 1,8$ D. Urošević, ${ }^{6,9}$ G. P. Rowell ${ }^{\oplus},{ }^{10}$ G. F. Wong, ${ }^{1,8}$ B.-Q. For, ${ }^{11,12}$ A. \\ N. O’Brien ${ }^{\oplus}, 1,5$ T. J. Galvin, ${ }^{1,5,13}$ L. Staveley-Smith ${ }^{\oplus},{ }^{11,12}$ R. P. Norris ${ }^{\odot}, 1,5$ T. Jarrett, ${ }^{14}$ \\ R. Kothes, ${ }^{15}$ K. J. Luken ${ }^{\oplus},{ }^{1,5}$ N. Hurley-Walker ${ }^{\oplus}{ }^{13}$ H. Sano ${ }^{\odot},{ }^{16}$ D. Onić, ${ }^{6}$ S. Dai, ${ }^{5}$ T. \\ G. Pannuti, ${ }^{17}$ N. F. H. Tothill, ${ }^{1}$ E. J. Crawford ${ }^{\oplus},{ }^{1}$ M. Yew, ${ }^{1}$ I. Bojičić, ${ }^{1}$ H. Dénes ${ }^{\oplus},{ }^{18}$ \\ N. McClure-Griffiths, ${ }^{19}$ S. Gurovich ${ }^{20}$ and Y. Fukui ${ }^{16}$
}

Affiliations are listed at the end of the paper

Accepted 2019 April 2. Received 2019 March 28; in original form 2018 October 26

\begin{abstract}
We report the discovery of a new Small Magellanic Cloud pulsar wind nebula (PWN) at the edge of the supernova remnant (SNR) DEM S5. The pulsar powered object has a cometary morphology similar to the Galactic PWN analogues PSR B1951+32 and 'the mouse'. It is travelling supersonically through the interstellar medium. We estimate the pulsar kick velocity to be in the range of $700-2000 \mathrm{~km} \mathrm{~s}^{-1}$ for an age between 28 and $10 \mathrm{kyr}$. The radio spectral index for this SNR-PWN-pulsar system is flat $(-0.29 \pm 0.01)$ consistent with other similar objects. We infer that the putative pulsar has a radio spectral index of -1.8 , which is typical for Galactic pulsars. We searched for dispersion measures up to $1000 \mathrm{~cm}^{-3} \mathrm{pc}$ but found no convincing candidates with an $\mathrm{S} / \mathrm{N}$ greater than 8 . We produce a polarization map for this $\mathrm{PWN}$ at $5500 \mathrm{MHz}$ and find a mean fractional polarization of $P \sim 23$ percent. The $\mathrm{X}$-ray power-law spectrum $(\Gamma \sim 2)$ is indicative of non-thermal synchrotron emission as is expected from PWN-pulsar system. Finally, we detect DEM S5 in infrared (IR) bands. Our IR photometric measurements strongly indicate the presence of shocked gas that is expected for SNRs. However, it is unusual to detect such IR emission in an SNR with a supersonic bow shock PWN. We also find a low-velocity H I cloud of $\sim 107 \mathrm{~km} \mathrm{~s}^{-1}$ that is possibly interacting with DEM S5. SNR DEM S5 is the first confirmed detection of a pulsar-powered bow shock nebula found outside the Galaxy.
\end{abstract}

Key words: radiation mechanisms: general - ISM: individual objects: DEM S5 - ISM: supernova remnants - Magellanic Clouds - radio continuum: ISM.

\section{INTRODUCTION}

The Small Magellanic Cloud (SMC) is a gas-rich irregular dwarf galaxy orbiting the Milky Way (MW). However, the proper motion of both the SMC and the Large Magellanic Cloud (LMC) is high, with Besla et al. (2007, and references therein) arguing that it could be on its first passage about the MW. With a current star formation rate (SFR) of $0.021-0.05 \mathrm{M}_{\odot} \mathrm{yr}^{-1}$ (For et al. 2018), it is ranked as

\footnotetext{
*E-mail: r.alsaberi@westernsydney.edu.au (RZEA); m.filipovic@westernsydney.edu.au (MDF)
}

the second nearest star-forming galaxy after the LMC to the MW. The relatively nearby distance of $\sim 60 \mathrm{kpc}$ (Macri et al. 2006) and the low Galactic foreground absorption $\left(N_{\mathrm{HI}} \sim 6 \times 10^{20} \mathrm{~cm}^{-2}\right)$ enables the entire source population in the SMC to be studied in X-rays down to a luminosity of $\sim 10^{33} \mathrm{erg} \mathrm{s}^{-1}$ (Haberl et al. 2012c). The recent star formation activity over the last $\sim 50 \mathrm{Myr}$ has created an environment where supernova remnants (SNRs) and X-ray pulsars are expected to be plentiful. In line with this, a large population of high-mass X-ray binaries (HMXBs) has been discovered and extensively studied by Haberl \& Sturm (2016). These objects are typically tens of millions of years old and have spin periods ranging 
from 1 to $1000 \mathrm{~s}$. However, the younger population of neutron stars (NSs) is still largely missing.

There are only two known young SMC pulsars: an 8.02 s anomalous X-ray pulsar, CXOU J010043.1-721134, with a characteristic age of $\sim 6700 \mathrm{yr}$ (Tiengo, Esposito \& Mereghetti 2008); the second one powers the only confirmed pulsar wind nebula (PWN) in the SMC, inside the large (74.5 pc diameter) SNR IKT 16. IKT 16 hosts a suspected pulsar with a spin-down luminosity $(\dot{E}) \sim 10^{37} \mathrm{erg} \mathrm{s}^{-1}$ and an age of $\sim 14.7 \mathrm{kyr}$ (Owen et al. 2011; Maitra et al. 2015). The PWN within IKT 16 was discovered with a dedicated Chandra observation, which resolved the hard (2-4.5 keV) X-ray point source seen with $X M M-N e w t o n$ into a faint and soft symmetric nebula surrounding a bright and hard point source. The symmetric morphology suggested that the PWN has not yet interacted with the reverse shock of the SNR. IKT 16 was also detected at radio continuum frequencies with a typical flat spectral energy distribution (SED) ${ }^{1}$ but without any indication of a point source coinciding with the $\mathrm{X}$-ray point source at the centre of the nebula.

Other well-studied SNRs in the SMC include SXP 1062 (Haberl et al. 2012a) that hosts a young Be X-ray binary pulsar with a long spin period in the centre of the remnant. Crawford et al. (2014) investigated the SNR HFPK 334 that has a prominent Xray point source close to the centre of the SNR. This X-ray source was considered to be a background object and not associated with the SNR. At the same time Roper et al. (2015) noted a lack of confirmed and prominent Type Ia SNRs, indicating that the sample of $\sim 25$ SNRs in the SMC may not be representative for any normal galaxy. However and recently, Maggi et al. (in preparation) list three likely Type Ia SNRs in the SMC.

Bozzetto et al. (2017) showed that only five PWNe (out of some 60 confirmed SNRs) are detected so far in the LMC. A further instance was investigated for a relationship between the LMC SNR J0529-6653 and the pulsar B0529-66 (Bozzetto et al. 2012, 2017) where the displacement between the midpoint (geometric centre) of the SNR to a pulsar candidate would be consistent with typical kick velocities. However, there was no radio frequency trail of the kind seen in other SNR/PWN systems.

Pulsars and their PWNe, moving supersonically through the ambient medium, are characterized by bow shaped shocks around the pulsar and/or cometary tails (Reynolds et al. 2017). This population is characterized by rotation-powered pulsars (RPPs) with ages between $10 \mathrm{kyr}$ and $3 \mathrm{Myr}$, with a spin-down power ( $\dot{E})$ ranging from $10^{33}$ to $10^{37} \mathrm{erg} \mathrm{s}^{-1}$ (Kargaltsev et al. 2017). Currently, there are $\sim 28$ known pulsars that show indications of supersonic motion (most with measured velocities). All of them reside in our Galaxy and exhibit a wide range of morphology (cf. Pavan et al. 2014a,b; Kargaltsev et al. 2017; Barkov, Lyutikov \& Khangulyan 2019). Although the LMC pulsar J0537-6910 (a.k.a. SNR N157 B or 30 Dor B) shows indications of some supersonic motion, its nature is yet to be firmly established. Similarly, LMC SNR N 206 (Klinger et al. 2002) shows an interesting linear tail-like feature but without an identifiable point source (pulsar) association. The other well-established PWNe in the LMC do not exhibit a bow shock morphology, but a typically flat radio SED is evident and located more or less centrally within the remnant (Haberl et al. 2012b).

DEM S5 (MCSNR J0041-7336; HFPK530) is among the largest SNRs in the SMC with a size of $245.5 \times 219.7$ arcsec $(71.4 \times 63.9 \mathrm{pc}$ at the distance of $60 \mathrm{kpc}$ to the SMC; see Fig. 1$)$, is X-ray faint, and has a complex shell like structure. The size of DEMS5 is measured from optical (MCELS; see Section 2.3) images while both X-ray and radio images show a smaller extent. With the present sensitivity (and resolution) of our X-ray and radio observations we cannot see the complete shell of SNR DEM S5, specifically, we are unable to measure any emission in the north and north-west region of the SNR. Therefore, the size of the complete shell can only be reliably measured from the optical Magellanic Cloud Emission Line Survey (MCELS) images. While lack of sensitivity may play an important role, this could also indicate that the outer regions seen in $[\mathrm{O} \mathrm{III}]$ as radiative shocks contains gas too cool to emit X-rays or radio continuum. A previous $X M M-$ Newton study of DEM S5 based on observations in which the source was substantially off-axis $(\sim 8$ arcmin) yielded poor constraints on the properties of the SNR due to statistical limitations (Filipović et al. 2008). However, an interesting outcome of the study was the detection of a soft X-ray source $\sim 67.5$ arcsec from the centre at $\mathrm{RA}(\mathrm{J} 2000)=00^{\mathrm{h}} 40^{\mathrm{m}} 47^{\mathrm{s}} .7$, and Dec. $(\mathrm{J} 2000)=-73^{\circ} 37^{\prime} 03^{\prime \prime}$. Filipović et al. (2008) noted then that the hard X-ray emission also coincided with the peak of the radio continuum image obtained with Australia Telescope Compact Array (ATCA).

Confirmation of the nature of this source is essential to refine our knowledge of pulsars in the SMC and more generally for stellar population studies of dwarf irregular galaxies. In particular, the detection of a new bow shock PWN would be of critical importance to study this small subsample of PWN systems. Furthermore, since the star formation history (SFH) and metallicity of the SMC is different from that of our Galaxy, it is an interesting system for studying the pulsar population, and comparing the younger population with the plentiful older ones (HMXBs).

In this paper, we present an analysis of radio and X-ray observational data of DEM S5 as follows: Section 2 details the radio X-ray and supplementary optical and infrared (IR) data; Sections 3 and 4, respectively, contain our results and a discussion. Finally, concluding remarks are given in Section 5.

\section{OBSERVATIONS AND DATA}

\subsection{Radio continuum observations}

We used ATCA observational data from projects CX310 and CX403 that were taken on the 2014 December 30 and 31; 2015 January 4, and 2017 December 22. These observations used the Compact Array Broadband Backend (CABB) (with $2048 \mathrm{MHz}$ bandwidth) in the $6 \mathrm{~A}$ and $6 \mathrm{C}$ array configuration, centred at wavelengths of $3 / 6 \mathrm{~cm}(v=4500-6500$ and $8000-10000 \mathrm{MHz}$; centred at 5500 and $9000 \mathrm{MHz})$, and $13 \mathrm{~cm}(v=2100 \mathrm{MHz})$. The observations were carried out in a frequency switching mode, totalling $\sim 444 \mathrm{~min}$ of integration at $4500-6500$ and $8000-10000 \mathrm{MHz}$ bands and $153 \mathrm{~min}$ of integration at $2100 \mathrm{MHz}$ frequency. Source PKS B1934638 was used as the primary (flux density) calibrator for the 2014 December 30, 2014 December 31, and 2015 January 4 data; and source PKS B0252-712 for the 2017 December 22 data. Source PKS B0230-790 was used as the secondary (phase) calibrator for all four observations (Table 1). The MIRIAD ${ }^{2}$ (Sault, Teuben \& Wright 1995) and KARMA ${ }^{3}$ (Gooch 1995) software packages were used to reduce and analyse the data. Images were formed using MIRIAD and the multifrequency synthesis tasks (Sault \& Wieringa 1994)

\footnotetext{
${ }^{2}$ http://www.atnf.csiro.au/computing/software/miriad/
}

${ }^{3}$ http://www.atnf.csiro.au/computing/software/karma/

${ }^{1}$ Defined as $S_{v} \propto v^{\alpha}$. 


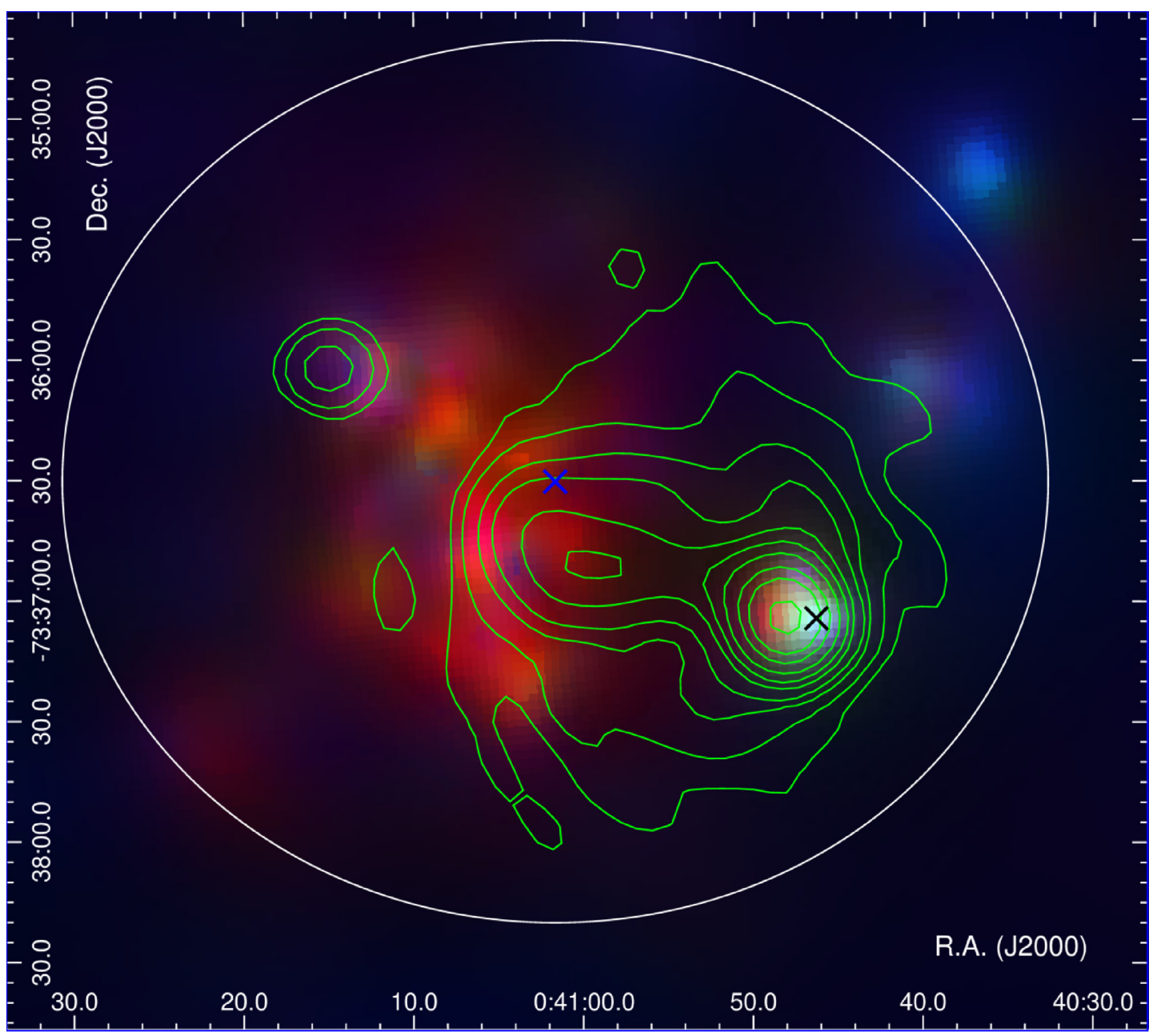

Figure 1. XMM-Newton EPIC RGB $(\mathrm{R}=0.2-1 \mathrm{keV}, \mathrm{G}=1-2 \mathrm{keV}, \mathrm{B}=2-4.5 \mathrm{keV})$ image of DEM S5. The optical size of the SNR and the centre are marked with a white ellipse $(245.5 \times 219.7$ arcsec extent) and blue cross, respectively (Filipović et al. 2008). The green contours denote the $1320 \mathrm{MHz}$ radio image. The contours are $0.5,1,2,3,5,7,10,15,20$, and $30 \mathrm{mJy} \mathrm{beam}^{-1}$ where we measure the local $\mathrm{rms}$ (or $1 \sigma$ ) to be $\sim 0.1 \mathrm{mJy} \mathrm{beam}^{-1}$. The beam size of the radio image is $16.3 \times 15.1 \mathrm{arcsec}$. The position of the hard X-ray point source $(2-4.5 \mathrm{keV})$ is marked with a black cross.

Table 1. Summary of ATCA observations for DEM S5.

\begin{tabular}{lccccccc}
\hline Date & $\begin{array}{c}\text { Project } \\
\text { code }\end{array}$ & $\begin{array}{c}\text { Array } \\
\text { configuration }\end{array}$ & $\begin{array}{c}\text { Integrated time } \\
(\mathrm{min})\end{array}$ & $\begin{array}{c}\text { Flux density } \\
\text { calibration }\end{array}$ & $\begin{array}{c}\text { Phase } \\
\text { calibration }\end{array}$ & $\begin{array}{c}\text { Frequency } \\
\text { Channels }\end{array}$ & $\begin{array}{c}\text { Bandwidth } \\
(\mathrm{MHz})\end{array}$ \\
\hline $2014-12-30$ & CX310 & 6A & 29.3 & $1934-638$ & $0230-790$ & 2049 & 5500,9000 \\
$2014-12-31$ & CX310 & 6A & 153 & $1934-638$ & $0230-790$ & 2049 & 2100 \\
$2015-01-04$ & CX310 & 6A & 37.3 & $1934-638$ & $0230-790$ & 2049 & 5500,9000 \\
$2017-12-22$ & CX403 & 6C & 377.3 & $0252-712$ & $0230-790$ & 2049 & 5500,9000 \\
\hline
\end{tabular}

therein with a Briggs weighting robust $=-1$ parameter for the $5500 \mathrm{MHz}$ image and 0 for the $2100 \mathrm{MHz}$ image. Both images were deconvolved by applying a primary beam correction. The same procedure was used for both the $Q$ and $U$ stokes parameter maps (see Fig. 2).

The $2100 \mathrm{MHz}$ image has a resolution of $3.7 \times 2.9 \mathrm{arcsec}$ with position angle $(\mathrm{PA})=58.1 \mathrm{deg}$, while the $5500 \mathrm{MHz}$ image had a resolution of $1.2 \times 0.89 \mathrm{arcsec}$ and $\mathrm{PA}=21.3 \mathrm{deg}$. Because of the low surface brightness and lack of short spacings, we were unable to create a reliable image at $9000 \mathrm{MHz}$ to sample the entire DEM S5 extended emission. However, we use the $9000 \mathrm{MHz}$ image for studying point sources within the field as they are largely not affected by the above shortcomings. Our new images at 2100,5500 , and $9000 \mathrm{MHz}$ have an rms noise of $0.1,0.013$, and $0.012 \mathrm{mJy} \mathrm{beam}^{-1}$, respectively.

We measured the integrated flux density of DEM S5 PWN from 11 separate images spanning a frequency range of $88-8640 \mathrm{MHz}$, which are summarized in Table 2. At the same time, we were able to measure the flux density from our two new $\mathrm{CABB}$ observations $(5500$ and $9000 \mathrm{MHz}$ ) of the two point-like sources in the field of DEMS5 which we associate with the putative pulsar and an unrelated background source $\left[\mathrm{RA}(\mathrm{J} 2000)=00^{\mathrm{h}} 41^{\mathrm{m}} 14.82\right.$, and Dec. (J2000) $\left.=-73^{\circ} 35^{\prime} 59^{\prime \prime} .9\right]$.

Similar to the studies of the radio continuum from the LMC by Bozzetto et al. (2017) and Maggi et al. (in preparation), we have used all available radio continuum data described in previous SMC 


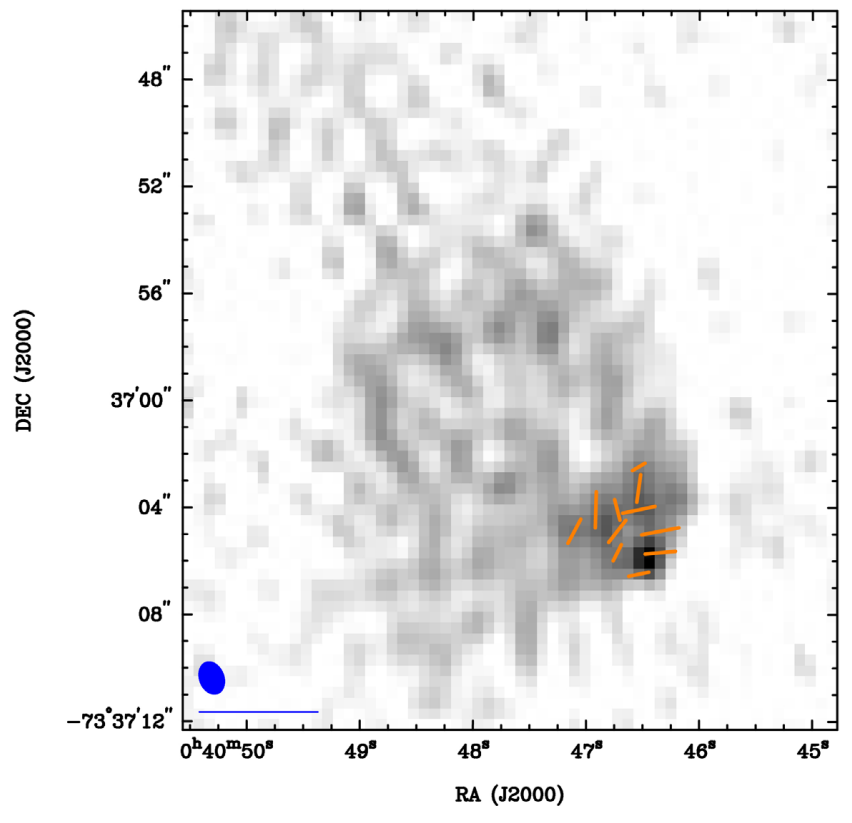

Figure 2. Fractional polarization vectors overlaid on the $5500 \mathrm{MHz}$ ATCA image of DEM S5 PWN. The blue ellipse in the lower left corner represents the synthesized beamwidth of $1.2 \times 0.89 \mathrm{arcsec}$ and the blue line below the ellipse represents a polarization vector of 100 per cent. We used robust $=-1$ weighting scheme to make this image. The peak fractional polarization value is $P=32 \pm 7$ per cent while the average polarization is measured to be $\sim 23$ per cent.

surveys (Filipović et al. 1997, 1998; Turtle et al. 1998; Filipović et al. 2002; Payne et al. 2004; Filipović et al. 2005b; Reid et al. 2006; Payne et al. 2007; Crawford et al. 2011; Wong et al. 2011a,b, 2012a,b). These data have been taken with the ATCA, Parkes, and the Molonglo Synthesis Telescope (MOST). Additionally, we make use of Murchison Widefield Array (MWA) data from For et al. (2018) and Joseph et al. (in preparation) obtained from the Australia Square Kilometre Array Pathfinder (ASKAP) Early Science Project observations of the SMC.

In Fig. 3, we show the new ATCA radio continuum images at 5500 and $2100 \mathrm{MHz}$ with the X-ray soft $(0.2-1.0 \mathrm{keV})$ and hard (2-4.5 keV) band contours overlaid, obtained from XMM-Newton. For all radio surveys (except the MWA), we used the MIRIAD task imf it in order to extract source integrated flux density, extensions [diameter/axes $(D)$ and PA]. Errors in flux density measurements predominately arose from uncertainties in defining the 'edge' of the remnant. However, we estimate these errors to be $<10$ per cent.

To estimate the flux density from the MWA surveys, we cut out $\approx 3 \times 3 \mathrm{deg}$ areas around the source from the GLEAM wideband extragalactic catalogue images (Hurley-Walker et al. 2017; For et al. 2018). ${ }^{4}$ Using the AEGEAN ToOLS package (Hancock et al. 2012; Hancock, Trott \& Hurley-Walker 2018), ${ }^{5}$ we ran the BACKGROUND AND NOISE ESTIMATOR and the AEGEAN sourcefinder on the $170-231 \mathrm{MHz}$ image to detect the SNR/PWNe. We then used the measured position as a prior and allowed the flux to vary when finding it in the $72-103,103-134$, and $139-170 \mathrm{MHz}$ images (priorized fitting). The errors on the integrated flux densities take into account the local rms, the fitting errors, and the overall flux scale calibration (13 percent at this low; Declination HurleyWalker et al. 2017). The source DEM S5 was unresolved at all MWA frequencies (as expected) so the integrated flux density is identical to the (background-subtracted) peak flux density.

\subsection{X-ray observations and analysis}

DEM S5 was observed serendipitously with XMM-Newton twice between 2006 and 2015 albeit at substantial off-axis angles. The observation details are given in Table 3. Fig. 1 displays the $X M M-$ Newton EPIC RGB image of DEM S5, showing the SNR in X-rays and a hard point-like $\mathrm{X}$-ray source shown with a black cross in the south-west direction at a distance of $21.2 \mathrm{pc}$ from the geometrical centre of the remnant. During both observations the point-like Xray source was located on a malfunctioning CCD of MOS1 and in the case of ObsID 0764780201, the source was also outside the readout area of the PN operated in small window mode. XMMNewton/EPIC (Strüder et al. 2001; Turner et al. 2001) observations were processed with the XMM-Newton, data analysis software SAS version 16.1.0. ${ }^{6}$

We searched for periods of high background flaring activity by extracting light curves in the energy range of 7.0 $15.0 \mathrm{keV}$ and removed the time intervals with background rates $\geq 8$ and $2.5 \mathrm{cts} \mathrm{ks}^{-1} \mathrm{arcmin}^{-2}$ for EPIC-PN and EPIC-MOS, respectively (Sturm et al. 2013). Events were extracted using the SAS task evselect by applying the standard filtering criteria (\#XMMEA_EP \&\& PATTERN $<=4$ for EPIC-PN and \#XMMEA_EM $\& \&$ PATTERN $<=12$ for EPIC-MOS).

In order to investigate whether the hard X-ray point-like source is extended, we extracted a radial profile centred on the source in the energy range of $1-2 \mathrm{keV}$ from the EPIC-PN image (ObsID 0301170301) as the signal-to-noise ratio peaks in this energy range. This was fitted with a Gaussian profile and the derived width was compared to that of the XMM-Newton point spread function (PSF) at the source position. The radial profile of the hard X-ray source is consistent with the PSF and we derived a $3 \sigma$ upper limit of 14 arcsec for the X-ray extent of the PWN. To further investigate the presence of a diffuse soft X-ray emission that may be associated with the source (and as may be indicated from Fig. 3, green contours), we performed the same exercise of comparison with the PSF in the energy range of $0.5-1 \mathrm{keV}$. An additional soft X-ray emission is detected peaking at $\sim 78$ arcsec, which originates from the X-ray SNR with its peak in emission at this distance (see X-ray image in Fig. 1).

We extracted the spectrum of the hard X-ray point source using a circular region of radius 20 arcsec centred around the source position obtained by Filipović et al. (2008). The background was chosen from two circular regions of radius 20 arcsec north and south of the source extraction region at a similar distance to the centre of the SNR. This should remove the contribution of soft X-ray emission from the SNR DEM S5, but given the irregular morphology of the SNR some uncertainty remains.

The SAS tasks rmfgen and arfgen were used to create the redistribution matrices and ancillary files for the spectral analysis. The spectrum was binned to achieve a minimum of one count per spectral bin. The spectral analysis was performed using the XSPEC fitting package, version 12.9 (Arnaud 1996) using the C-statistic. Errors were estimated at 90 per cent confidence intervals. The spectra from

\footnotetext{
${ }^{4}$ http://gleam-vo.icrar.org

${ }^{5}$ https://github.com/PaulHancock/Aegean
}

${ }^{6}$ Science Analysis Software (SAS): http://xmm.esac.esa.int/sas/. 
Table 2. Measurements of integrated flux density of DEM S5 PWN and putative pulsar as well as the unrelated background source found within the field of DEM S5. Because of the poor survey resolution and sensitivity we could not detect or measure the flux density of the pulsar and background source at MWA frequencies.

\begin{tabular}{|c|c|c|c|c|c|}
\hline$v(\mathrm{MHz})$ & PWN $S_{v}(\mathrm{Jy})$ & Pulsar $S_{v}(\mathrm{Jy})$ & Background $S_{v}(\mathrm{Jy})$ & Telescope & Reference \\
\hline 118 & $0.256 \pm 0.052$ & & & MWA & For et al. (2018) \\
\hline 155 & $0.234 \pm 0.040$ & & & MWA & For et al. (2018) \\
\hline 200 & $0.205 \pm 0.033$ & & & MWA & For et al. (2018) \\
\hline 843 & $0.150 \pm 0.015$ & & $0.0044 \pm 0.0005$ & MOST & $\begin{array}{l}\text { Maggi et. al. (in } \\
\text { preparation) }\end{array}$ \\
\hline 960 & $0.138 \pm 0.014$ & & $0.0038 \pm 0.0004$ & ASKAP & $\begin{array}{l}\text { Joseph et. al. (in } \\
\text { preparation) }\end{array}$ \\
\hline 1420 & $0.128 \pm 0.013$ & & & ATCA & $\begin{array}{l}\text { Maggi et. al. (in } \\
\text { preparation) }\end{array}$ \\
\hline 2100 & - & $0.003 \pm 0.001$ & $0.0017 \pm 0.0002$ & ATCA & This work \\
\hline 2370 & $0.114 \pm 0.011$ & & & ATCA & $\begin{array}{l}\text { Maggi et. al. (in } \\
\text { preparation) }\end{array}$ \\
\hline $\begin{array}{l}9000 \\
\alpha \pm \Delta \alpha\end{array}$ & $\begin{array}{c}- \\
-0.29 \pm 0.01\end{array}$ & $\begin{array}{c}0.00022 \pm 0.00007 \\
-1.8 \pm 0.2\end{array}$ & $\begin{array}{c}0.00065 \pm 0.00007 \\
-0.81 \pm 0.04\end{array}$ & ATCA & This work \\
\hline
\end{tabular}

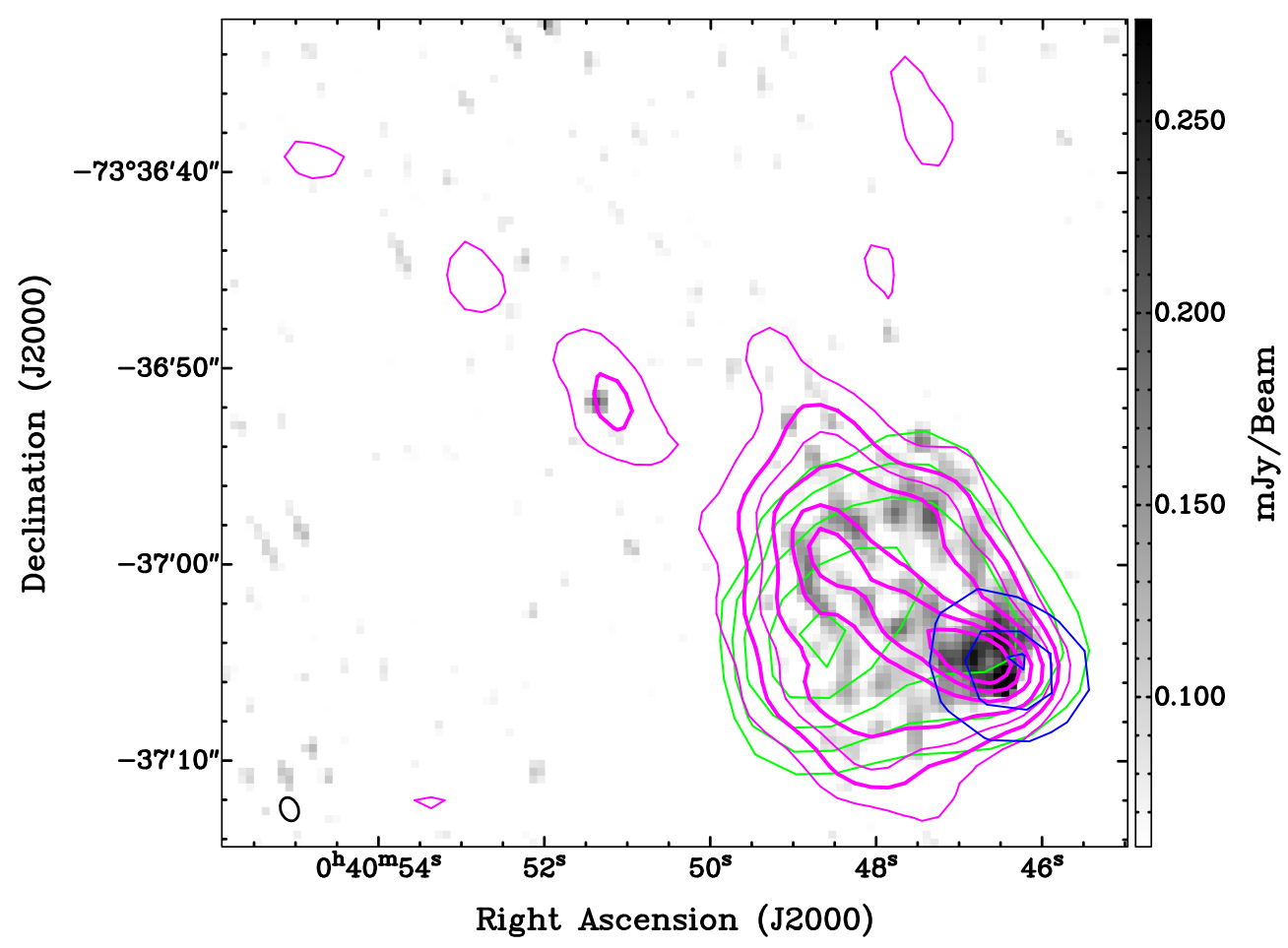

Figure 3. A zoomed in image of the point-like source (pulsar) and PWN within DEM S5 showing a comparison between the radio and X-ray wavelengths. The grey-scale image is from the ATCA observation at $5500 \mathrm{MHz}$, magenta contours are from the $2100 \mathrm{MHz}$ image $\left(0.3,0.5,0.7,1,1.5,2\right.$, and $\left.^{-5} .5 \mathrm{mJy}^{\mathrm{beam}}{ }^{-1}\right)$; green contours are from XMM-Newton $\left(0.2-1 \mathrm{keV} ; 1.7,1.9,2.1,2.3\right.$, and $2.5 \times 10^{-5} \mathrm{cts} \mathrm{s}^{-1}$ pixel $\left.^{-1}\right)$; and blue contours are the XMM-Newton $(2-4.5 \mathrm{keV}$; levels are $1.1,1.3$, and $1.4 \times 10^{-5} \mathrm{cts} \mathrm{s}^{-1}$ pixel $^{-1}$ ). The black ellipse in the lower left corner represents the synthesized beamwidth of $1.2 \times 0.89$ arcsec. An rms noise at $5500 \mathrm{MHz}$ image is 0.013 and $0.1 \mathrm{mJy}^{-1}$ beam ${ }^{-1}$ at $2100 \mathrm{MHz}$ image. 
Table 3. XMM-Newton observations of the hard X-ray point source inside DEM S5.

\begin{tabular}{|c|c|c|c|c|c|}
\hline $\begin{array}{l}\text { Date } \\
\text { yyyy } / \mathrm{mm} / \mathrm{dd}\end{array}$ & ObsID & $\begin{array}{c}\text { Exposure } \\
\text { PN/MOS2 } \\
\text { (ks) }\end{array}$ & $\begin{array}{l}\text { CCD readout mode } \\
\text { PN/MOS2 }\end{array}$ & $\begin{array}{l}\text { Off-axis angle } \\
\text { PN/MOS2 }\end{array}$ & $\begin{array}{l}\text { Telescope } \\
\text { vignetting } \\
\text { PN/MOS2 }\end{array}$ \\
\hline 2006/06/04 & 0301170301 & $15.5 / 21.1$ & Full frame/full frame & & $0.61 / 0.65$ \\
\hline $2015 / 10 / 16$ & 0764780201 & $-/ 47.9$ & Small window/full frame & $\begin{array}{l}8.4 \mathrm{arcmin} / 7.4 \operatorname{arcmin} \\
-/ 9.7 \mathrm{arcmin}\end{array}$ & $-/ 0.53$ \\
\hline
\end{tabular}

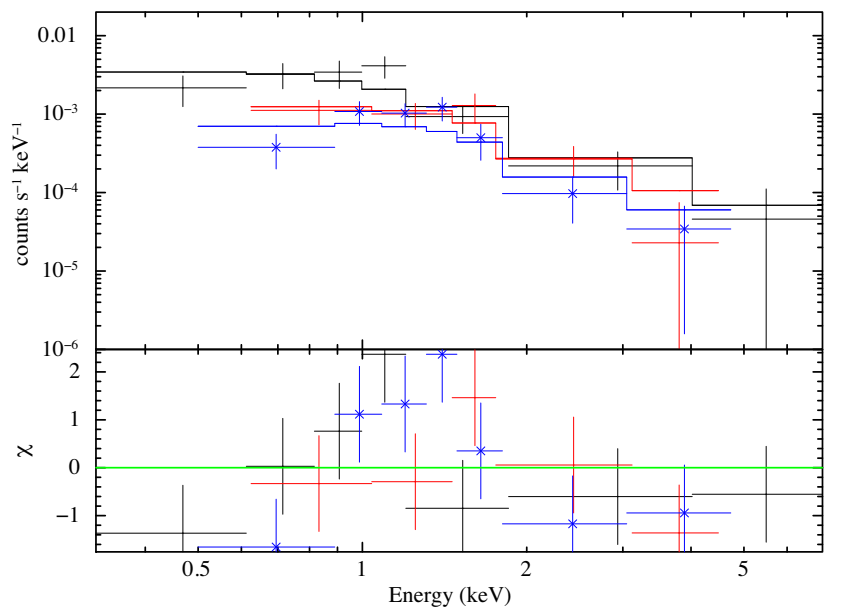

Figure 4. The upper panel shows the simultaneous spectral fit using spectra from all the available XMM-Newton/EPIC cameras (PN: ObsID 0301170301 in black, MOS2: ObsID 0301170301 in red and MOS2: ObsID 0764780201 in blue double crosses) along with the best-fitting model. The lower panel displays the residuals after the fit. The plots have been rebinned for visual clarity.

Table 4. X-ray spectral parameters of the point-like source in DEM S5. Absorption $^{a}$ was fixed to $6 \times 10^{20} \mathrm{~cm}^{-2}$. Errors are quoted at $90 \%$ confidence.

\begin{tabular}{lc}
\hline Parameters & Value \\
\hline$\Gamma$ & $1.99_{-0.28}^{+0.26}$ \\
Flux $^{b}(0.5-8.0 \mathrm{keV})$ & $3.1 \pm 0.9$ \\
Flux (unabsorbed) ${ }^{b}(0.3-8.0 \mathrm{keV})$ & $3.6 \pm 1.0$ \\
X-ray luminosity at $d_{\mathrm{smc}}=60 \mathrm{kpc}\left(\mathrm{erg} \mathrm{s}^{-1}\right)$ & $1.5 \times 10^{34}$ \\
X-ray luminosity at $d_{\mathrm{smc}}=67.5 \mathrm{kpc}\left(\mathrm{erg} \mathrm{s}^{-1}\right)$ & $1.9 \times 10^{34}$ \\
\hline
\end{tabular}

Notes. ${ }^{a}$ Absorption was fixed equal to the Galactic value along the line of sight of SMC to $N_{\text {Hgal }}=6 \times 10^{20} \mathrm{~cm}^{-2}$.

${ }^{b}$ Flux in units of $10^{-14} \mathrm{erg} \mathrm{cm}^{-2} \mathrm{~s}^{-1}$.

both observations were fitted simultaneously with absorbed singlecomponent emission models. Given the low statistics of the spectra power law (photon index $\Gamma \sim 2$ ), bremsstrahlung (temperature $k T$ $\sim 1.7 \mathrm{keV}$ ), and APEC plasma emission ( $k T \sim 2.1 \mathrm{keV}$ ) yield formally indistinguishable fit quality ( $\chi^{2}$ values of $121.2,121.1$, and 119.9, respectively, for 185 degrees of freedom). The spectra with bestfitting absorbed power-law model is shown in Fig. 4. The index of the emitting particles ( $p$ ) has been calculated by using the equation $p=\Gamma \times 2-1$, where $\Gamma$ is the index of the (synchrotron) energy spectrum. The $p$ value is consistent with the expected range of $1 \leq p \leq 3$ for non-thermal emission. The spectral parameters are tabulated in Table 4. No excess absorption was required apart from the Galactic value along the line of sight to the SMC. This is in further support of the source being located at the near side

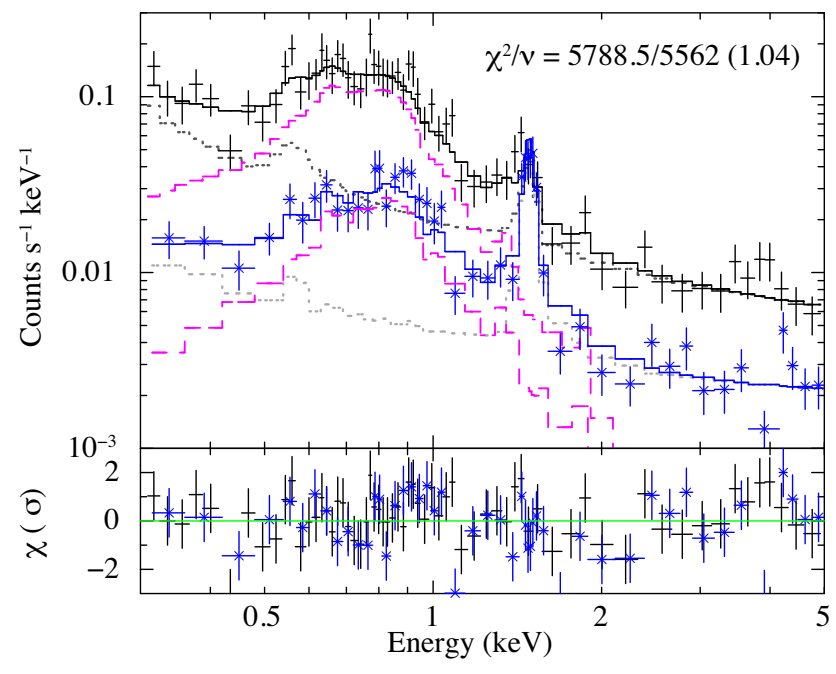

Figure 5. X-ray spectra of the diffuse emission of SNR DEM S5. Colours for data are the same as in Fig. 4, but MOS data for ObsID 0301170301 have been omitted for the sake of clarity. The total background models for PN and MOS are shown by the dotted grey lines. The SNR emission models are shown by the dashed magenta lines, as convolved with the PN and MOS spectral responses (top and bottom curves, respectively). The lower panel displays the residuals.

of the SMC and argues against the source being a background object, as the absorption at the source position through the depth of the SMC is $N_{\mathrm{HI}} \sim 4 \times 10^{21} \mathrm{~cm}^{-2}$ (Stanimirovic et al. 1999). The value of $\Gamma$ is consistent with typical X-ray spectral slopes observed in Kargaltsev \& Pavlov (2008) as is expected from nonthermal synchrotron emission originating from a PWN/pulsar. We further verified that the X-ray spectral shape and the flux were stable between the two XMM-Newton observations separated by 8 yr by setting the $\Gamma$ and normalizations free between the observations. A non-varying spectrum is in further support of a PWN (containing a putative pulsar unresolved in the current observations) origin of the source. The detection of a hard X-ray point source with $X M M$ Newton ascertains the fact that we are unable to further resolve the PWN/pulsar composite, and require a higher spatial resolution on-axis Chandra observation for the purpose.

In parallel, we extracted spectra for the diffuse X-ray emission in the east part of the SNR. For such faint extended emission we did not subtract background emission, but rather modelled all sources of background directly in the analysis and added a model for the diffuse emission on top of it. The method we used has been described extensively for XMM-Newton in Maggi et al. (2016). Even though we included MOS2 data from a second XMM-Newton observation (Table 3) not available in the first analysis of Filipović et al. (2008), the analysis is still hampered by a relatively short exposure time and off-axis location of the source. 
The spectrum of the diffuse emission, shown in Fig. 5, is obviously thermal, typical for shock-heated plasma in SNRs. We fit the spectrum with a non-equilibrium ionization shock model with variable abundances (vpshock in XSPEC), finding a temperature $k T=$ $0.65_{-0.12}^{+0.09} \mathrm{keV}$ and ionization age $n_{e} t=2.2\left({ }_{-0.9}^{+1.3}\right) \times 10^{11} \mathrm{~s} \mathrm{~cm}^{-3}$. When let free, the abundances of the main elements $(\mathrm{O}, \mathrm{Ne}, \mathrm{Mg}, \mathrm{Fe}$, and $\mathrm{Si}$ ) do not depart significantly from the SMC ISM abundance pattern (Russell \& Dopita 1992). With an observed flux in the 0.5$8 \mathrm{keV}$ band of $(7.6 \pm 0.5) \times 10^{-14} \mathrm{erg} \mathrm{cm}^{-2} \mathrm{~s}^{-1}$ absorption-corrected flux of $\left(1.01_{-0.15}^{+0.40}\right) \times 10^{-13} \mathrm{erg} \mathrm{cm}^{-2} \mathrm{~s}^{-1}$, DEM S5 is in the faint end of the SMC SNR population (e.g. Maggi et al. 2016). The bestfitting absorption column (on top of the Galactic one) is relatively low $\left(N_{\mathrm{H}}=1.1 \times 10^{21} \mathrm{~cm}^{-2}\right)$ and at the $3 \sigma$ level consistent with 0 , as for the PWN candidate. Therefore, from the point of view of absorption, both the hard X-ray source and the diffuse emission are consistent within one another.

Naively, the shape and location of this emission would suggest that it is thermal emission from the supernova ejecta that was shocked and heated by the reverse shock. However, this is not sustained by the data, which are fully consistent with SMC abundances, suggesting instead a shocked ISM origin. It remains possible that shock-heated ejecta contribute to the diffuse emission, at a level low enough not to be significantly detected in the available XMMNewton data.

\subsection{Optical and infrared observations}

The MCELS was carried out at the $0.6 \mathrm{~m}$ University of Michigan/CTIO Curtis Schmidt telescope, equipped with a SITE $2048 \times$ $2048 \mathrm{CCD}$, which gave a field of $1.35 \times 1.35 \mathrm{deg}$ at a scale of $2.4 \times 2.4$ arcsec pixel $^{-1}$ (Smith, Leiton \& Pizarro 2000; Pellegrini et al. 2012). The SMC was mapped in narrow bands corresponding to $\mathrm{H} \alpha$, [O III] $(\lambda=5007 \AA)$, and [S II] $(\lambda=6716,6731 \AA)$, plus matched red and green continuum bands. All the data have been continuum subtracted, flux-calibrated, and assembled into mosaic images. A cut-out around the area of DEM S5 can be seen in Fig. 6.

We also make use of SMC observations from Spitzer SAGE (Surveying the Agents of a Galaxy's Evolution; Gordon et al. 2011). Specifically, we used four IRAC bands: band-1 $(3.6 \mu \mathrm{m})$, band-2 $(4.5 \mu \mathrm{m})$, band-3 $(5.8 \mu \mathrm{m})$, and band-4 $(8.0 \mu \mathrm{m})$, as well as MIPS at $24 \mu \mathrm{m}$.

\subsection{H I observations}

Observations of $\mathrm{H}$ I have previously been carried out over the whole SMC (20 deg $\left.{ }^{2}\right)$ by Stanimirovic et al. (1999) using the ATCA $375 \mathrm{~m}$ configuration and the Parkes telescopes. The angular resolution was 98 arcsec, and velocity resolution was $1.65 \mathrm{~km} \mathrm{~s}^{-1}$, with a column density sensitivity of $4.2 \times 10^{18} \mathrm{~cm}^{-2}$ in the same velocity interval. The heliocentric reference frame was used to define velocities.

Recent ASKAP data are also available over a similar field of view, but at the higher angular resolution of $27 \times 35$ arcsec and the lower velocity resolution of $3.9 \mathrm{~km} \mathrm{~s}^{-1}$ (McClure-Griffiths et al. 2018). The column density sensitivity per $3.9 \mathrm{~km} \mathrm{~s}^{-1}$ channel is similar to the ATCA data at $5.2 \times 10^{18} \mathrm{~cm}^{-2}$.

\section{RESULTS}

\subsection{PWN and compact source inside the SNR DEM S5}

We detect a point-like source at $5500 \mathrm{MHz}$, which we consider likely to be a pulsar, at RA $(J 2000)=00^{\mathrm{h}} 40^{\mathrm{m}} 46^{\mathrm{s}} .49$ and Dec. $(\mathrm{J} 2000)=-$ $73^{\circ} 37^{\prime} 5^{\prime \prime} .8$, with a trail/jet behind it and a nebulosity that is morphologically indicative of a PWN (Fig. 3). This trail is clearly visible only in our 2100 and $1320 \mathrm{MHz}$ images because of the sensitivity and missing short spacings at higher frequencies. One should also consider that this trail might be a so-called relic PWN behind the present putative pulsar similar to Galactic SNR/PWN G5.4-1.2 (Kothes 2017). While somewhat unlikely, as the trail is still part of PWN system (see Fig. 1), only future higher sensitivity and resolution radio continuum observations may reveal true nature of this possibility.

The position of the radio point source is also coincident with the hard X-ray point source. There seems to be a small displacement $(<2$ arcsec) between the centremost blue $(2-4.5 \mathrm{keV}) \mathrm{X}$-ray contour and the radio point-like source. This is attributed to the fact that the X-ray counterpart of the putative pulsar cannot be separated from the PWN/pulsar composite with the current XMM-Newton observations and hence its accurate X-ray position cannot be ascertained. Fig. 3 further shows that the PWN emission close to the pulsar is dominated by hard X-rays. This is due to the fact that the $\mathrm{X}$-ray synchrotron electrons have a much shorter lifetime than radio ones and the X-ray synchrotron spectrum from the PWN would get steeper when moving further away from the pulsar.

The measured integrated flux density of the point source (putative pulsar) at 5500 and $9000 \mathrm{MHz}$ is $0.42 \mathrm{mJy}(\sim 14 \sigma)$ and $<0.22 \mathrm{mJy}$ $(\sim 3 \sigma)$, respectively. At $2100 \mathrm{MHz}$, we cannot precisely measure flux density, but there is a strong indication of an order of magnitude stronger integrated flux density (around $3 \mathrm{mJy}$, see Table 2). This would make the SED of this point source (putative pulsar) very steep with a spectral index of $\alpha \sim-1.8$. The SED of most known Galactic pulsars can be described above $100 \mathrm{MHz}$ by a simple power law with a mean spectral index of $\sim-1.8$ (Han et al. 2016), with a range from -0.46 to -4.84 . Therefore, we propose that this point source is most likely the compact remnant (pulsar or a fast spinning NS) of the DEM S5 supernova explosion with its associated PWN. Also, ultra steep spectrum and compact steep spectrum (CSS) sources would have such a steep radio spectrum (Collier et al. 2016). However, as we see the nebulosity behind the point source, it is very unlikely to be CSS with jet active galactic nuclei (AGNs) like structure.

Figs 1, 3, and 7 show trail-like emission from PWN with the length of $\sim 37 \operatorname{arcsec}(\sim 10.8 \mathrm{pc})$. The direction of the trail is pointing towards the geometric centre of the SNR $\left[\mathrm{RA}(\mathrm{J} 2000)=00^{\mathrm{h}} 41^{\mathrm{m}} 01^{\mathrm{s}} .675\right.$ and Dec. $\left.(\mathrm{J} 2000)=-73^{\circ} 36^{\prime} 30^{\prime \prime} \cdot 38\right]$. We also note that the putative pulsar (compact source) is 'leading' the conically shaped PWN that trail behind it. This is a strong indication that the putative pulsar is moving away from the SNR explosion site at a supersonic velocity (see Table 5). Morphologically, this would qualify the DEM S5 system (pulsar and PWN) as a 'rifle bullet' type (Barkov et al. 2019) where the spin and velocity are aligned. The most likely Galactic bow shock PWN analogue would be PSR B1951+32 in SNR CTB 80 (see Safi-Harb, Ogelman \& Finley 1995; Moon et al. 2004) and 'mouse' PWN (J1747-2956) (Gaensler et al. 2004; Klingler et al. 2018) where a similar morphology is detected. However, according to Kargaltsev et al. (2017) PSR B1951+32 does not move supersonically. Fig. 7 clearly shows that the putative pulsar with its PWN is reaching the edge of the optical remnant. The putative pulsar may be even outside of it, if the motion is not entirely perpendicular to the line of sight. Although purely visually, it would be probably just at the edge. The densities outside and inside the SNR should be different, with outside expected to be much higher. Therefore, we expect the PWN to be somewhat narrower with higher ambient density. 


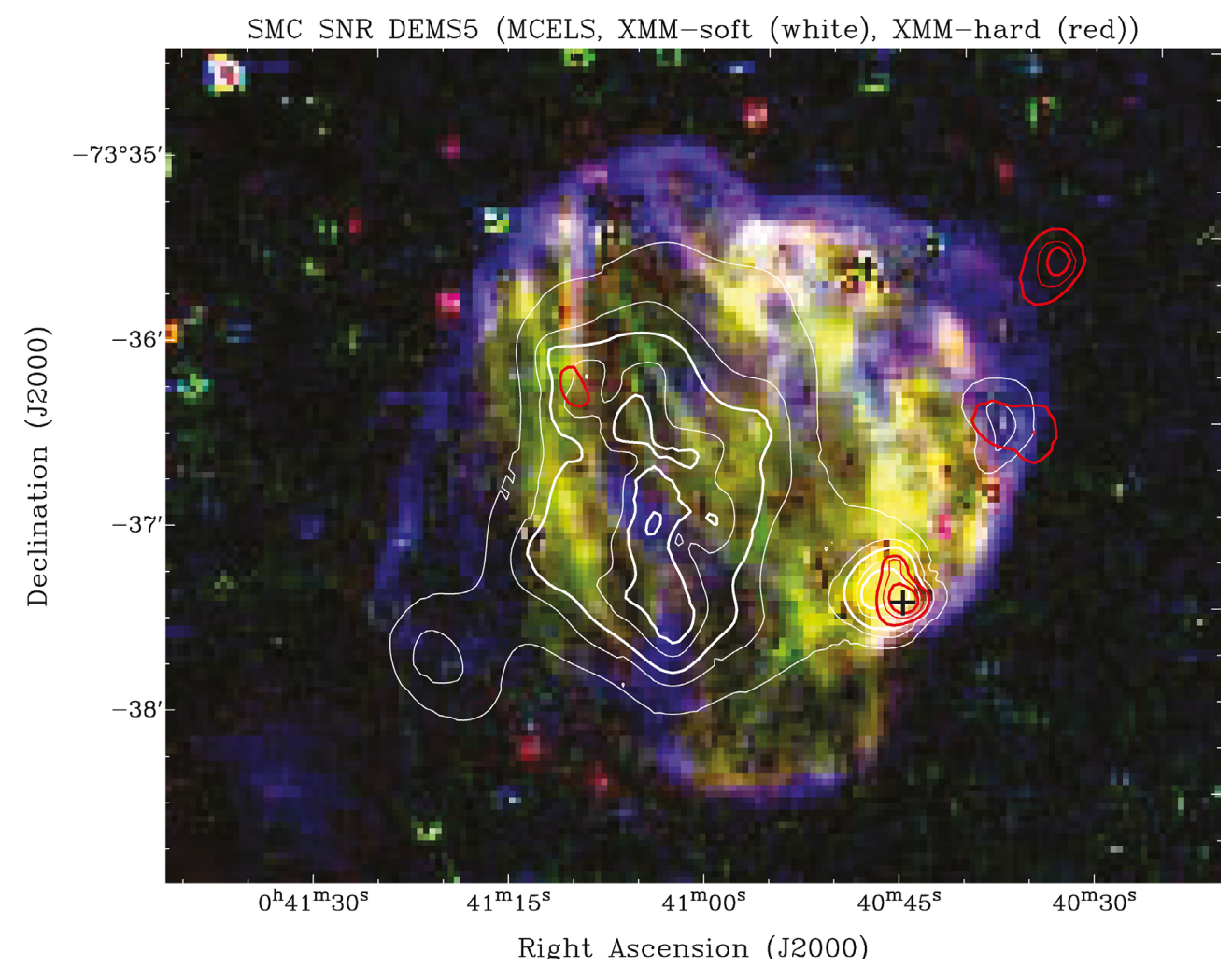

Figure 6. MCELS composite image of DEM S5 (RGB $=\mathrm{H} \alpha$, [S II], [O III]) overlaid with XMM-Newton EPIC contours, soft $(0.2-1 \mathrm{keV})$ in white (levels are $0.5,0.75,1,1.5$, and $2 \times 10^{-5} \mathrm{cts} \mathrm{s}^{-1}$ pixel $^{-1}$ ) and hard (2-4.5 keV) in red (levels are $0.5,0.75$, and $1 \times 10^{-5} \mathrm{cts} \mathrm{s}^{-1}$ pixel $^{-1}$ ). The position of the suggested pulsar is marked with a black plus sign.

In Table 5, we used various SNR ages (from 5 to $50 \mathrm{kyr}$ ) to estimate the kick (transverse) velocity of the pulsar. We also varied the distance to DEM S5 as Scowcroft et al. (2016) suggested that at the western end of the SMC might reach up to $67.5 \mathrm{kpc}$. The observed distance between the radio point-like source (putative pulsar) and the centre of the SNR is measured to be $\sim 73 \operatorname{arcsec}$ (or $21.2 / 23.9 \mathrm{pc}$ at the $60 / 67.5 \mathrm{kpc}$ distance respectively and assuming motion to be perpendicular to the line of sight).

The kick (transverse) velocities in other similar PWN bow shock systems are estimated to be in range of $60-2000 \mathrm{~km} \mathrm{~s}^{-1}$ (see their table 1, Kargaltsev et al. 2017) for the pulsar through the ambient medium. We take this velocity as a possible upper limit which then indicates a very high DEM S5 pulsar velocity through the ambient medium given that the minimum age would be in order of $>10 \mathrm{kyr}$ (see Table 5). Other constraints on the age of DEM S5 come from the IKT 16 PWN/SNR system that has an estimated age of $14.7 \mathrm{kyr}$ and has a somewhat larger SNR diameter of $74.5 \mathrm{pc}$ versus $67.6 \mathrm{pc}$ for DEM S5 indicating that IKT $16 \mathrm{PWN} / \mathrm{SNR}$ is expanding at a somewhat lower rate probably because the local environment is denser than in/around DEM S5 $\left(N_{\mathrm{H} \mathrm{I}} \sim 4 \times 10^{21} \mathrm{~cm}^{-2}\right.$ for DEM S5 versus $N_{\mathrm{HI}} \sim 6 \times 10^{21} \mathrm{~cm}^{-2}$ for IKT 16). However, one should be cautious about the line-of-sight column density, calculated by integrating along several $\mathrm{kpc}$ for the SMC because of variations in the local ambient medium density. This would suggest that the age of DEM S5 PWN is between 10 and $15 \mathrm{kyr}$ with kick velocities of $\sim 1500-2500 \mathrm{~km} \mathrm{~s}^{-1}$. Also, Reynolds et al. (2017) suggest that the pulsar would eventually leave the SNR at an age of $\sim 20-200 \mathrm{kyr}$ that further limits our DEM S5 PWN as it is still within the SNR boundaries. Given a lower age limit of $\sim 10 \mathrm{kyr}$, the kick-off velocity of $\sim 2000 \mathrm{~km} \mathrm{~s}^{-1}$ is derived using its distance from the DEM S5 geometric centre, which is the most likely explosion site in a uniform ISM. If the ISM is inhomogeneous or has a large-scale gradient the explosion site would be shifted $(\sim 4.5 \operatorname{arcsec}$ or $1.3 \mathrm{pc})$ from the geometrical centre towards the west [at RA $(\mathrm{J} 2000)=00^{\mathrm{h}} 41^{\mathrm{m}} 01^{\mathrm{s}} .53$ and Dec.(J2000) $=-73^{\circ} 36^{\prime} 26^{\prime \prime}$. 6 ; with ellipse radius of $\sim 34 \times$ $22 \mathrm{pc})$. In any case, a large kick velocity would certainly still need to be accounted for. We emphasize that the age and size of different SNRs might not be appropriate to compare as they all come with their own unique characteristics.

The fastest known moving pulsars are 'Morla' (J0357+3205) (Marelli et al. 2013; Kargaltsev et al. 2017) and 'Lighthouse' (Pavan et al. 2014a,b; Kargaltsev et al. 2017) at $2000 \mathrm{~km} \mathrm{~s}^{-1}$. We note that our DEM S5 PWN cometary tail has a conical shape with the angle behind the putative pulsar of $\sim 75-80 \mathrm{deg}$, which suggests somewhat slower velocity than $\sim 2000 \mathrm{~km} \mathrm{~s}^{-1}$ that we estimate above; unless our PWN system is not just characterized as 'rifle bullet' but also 'frisbee' or 'cart-wheel' (Barkov et al. 2019). Additionally, HollandAshford et al. (2017) and Katsuda et al. (2018) suggested that pulsars move preferentially opposite to the ejecta, which lends support to the interpretation that the diffuse X-ray emission originates from shock-heated ejecta.

DEM S5 is a very large SNR in general, which argues for a late stage of evolution. We use Cioffi, McKee \& Bertschinger (1988) for calculations of radiative SNRs. Using their equations for the onset of the pressure-driven snowplow or radiative phase, we obtain a minimum ambient density of $0.35 \mathrm{~cm}^{-3}$ and a lower limit for the age of $24 \mathrm{kyr}$, using $22 \mathrm{pc}$ radius (towards the west) and $10^{51} \mathrm{erg}$ for the explosion energy - certainly nothing unusual. For a density of $1 \mathrm{~cm}^{-3}$ the age would be about $48 \mathrm{kyr}$ but the age estimate could be even a lot higher. We also need to consider the amount of material 


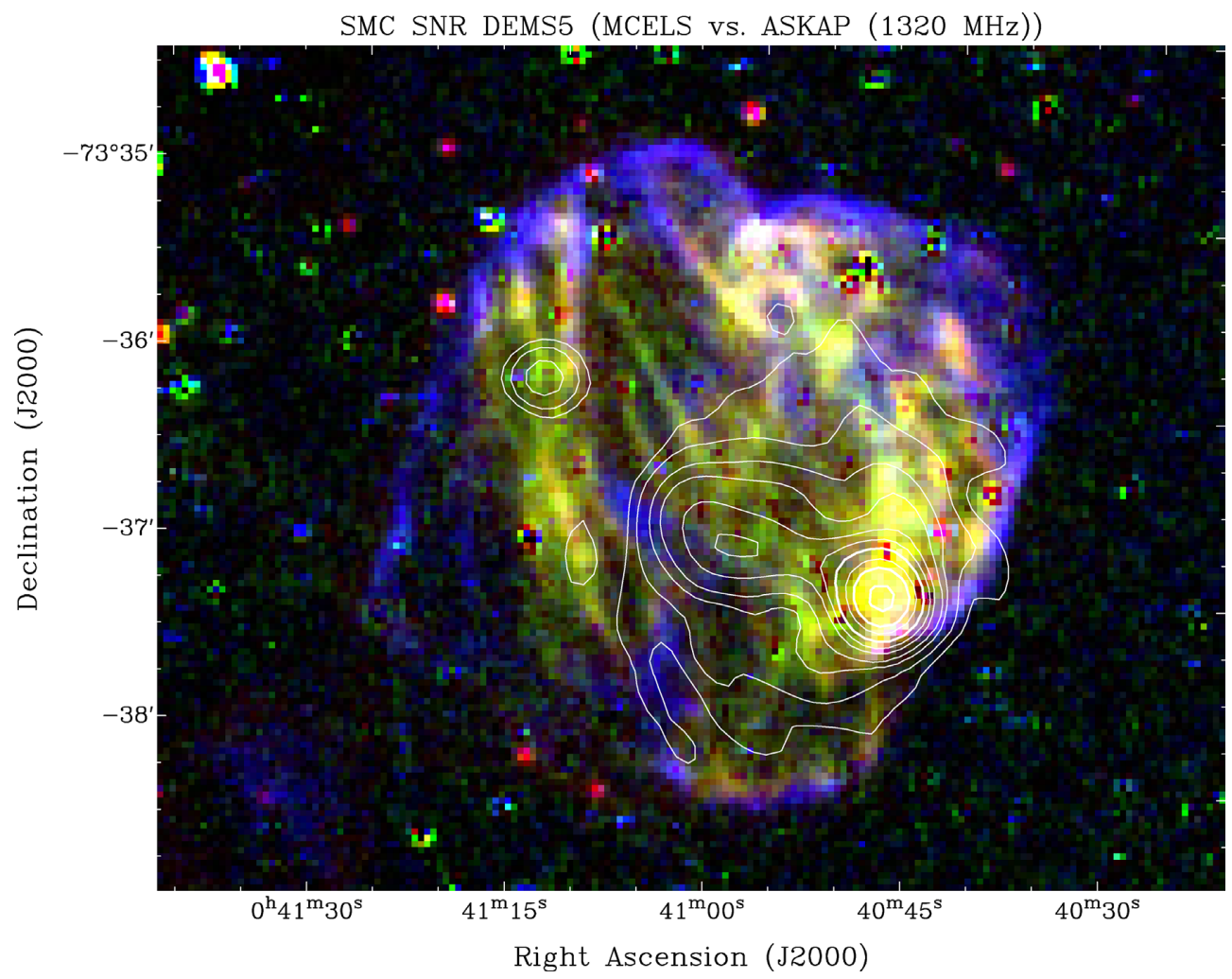

Figure 7. MCELS composite image of DEM S5 [HFPK 530] (RGB = H $\alpha$, [S II], [O III]) overlaid with ASKAP contours (white solid) at 1320 MHz. The radio image contours are as same as in Fig. 1. An obvious radio continuum tail-like feature is clearly pointing towards the geometric centre of DEM S5. We also note a point source north-east from the remnant centre that we classify as a most likely background galaxy.

Table 5. Derived pulsar kick velocity for the DEM S5 progenitor: estimated as a function of SNR age $\left(V_{\mathrm{psr}}=2 \times d_{\text {smc }} \times \mathrm{TAN}(\theta / 2) /\right.$ age $)$ that simplifies to $\left(\theta \times d_{\text {smc }}\right) /$ age since $\theta \ll 1$. The angular distance is $\theta=73$ arcsec. The adopted distance of $d_{\mathrm{smc}}=67.5 \mathrm{kpc}$ for the western side of the SMC is suggested by Scowcroft et al. (2016).

\begin{tabular}{lcc}
\hline $\begin{array}{l}\text { SNR age } \\
(\mathrm{kyr})\end{array}$ & $\begin{array}{c}V_{\text {psrat 60 kpc }} \\
\left(\mathrm{km} \mathrm{s}^{-1}\right)\end{array}$ & $\begin{array}{c}V_{\text {psrat } 67.5 \mathrm{kpc}} \\
\left(\mathrm{km} \mathrm{s}^{-1}\right)\end{array}$ \\
\hline 5.0 & 4157 & 4676 \\
7.5 & 2771 & 3117 \\
10.0 & 2078 & 2338 \\
12.5 & 1663 & 1870 \\
15.0 & 1386 & 1559 \\
17.5 & 1188 & 1336 \\
20.0 & 1039 & 1169 \\
22.5 & 924 & 1039 \\
25.0 & 831 & 935 \\
30.0 & 693 & 779 \\
40.0 & 520 & 584 \\
50.0 & 416 & 468 \\
\hline
\end{tabular}

that would have been swept up in such a large volume if we have normal densities. For this to still be a Sedov SNR we would need very low densities to make this still applicable. The largest distance between the most likely location of the supernova explosion and the edge of the SNR - as indicated by the [O III] shell - is almost straight to the east. In between, we find the diffuse X-ray emission, which might be including some shock-heated ejecta. In this case, the reverse shock must have pass this area that stretches all the way to the centre of the SNR. With an assumed ejecta mass of 10 solar masses, a radius of $40 \mathrm{pc}$ and the canonical value for the explosion energy of $10^{51} \mathrm{erg}$, we require an ambient density of $0.02 \mathrm{~cm}^{-3}-$ if the reverse shock just reached the location of the $\mathrm{SN}$ (see their equations McKee \& Truelove 1995). This is the typical density in the interior of a stellar wind bubble (e.g. Weaver et al. 1977). With these parameters the age would be $28 \mathrm{kyr}$ and the PWN/pulsar system kick-off velocity would decrease to a 'reasonable' 700 $800 \mathrm{~km} \mathrm{~s}^{-1}$. We note that the ejecta mass is not very critical here. If we insert this into the equations of Cioffi et al. (1988) we get an ambient density of $0.45 \mathrm{~cm}^{-3}$ for the right (west) part of the SNR. Therefore, this scenario seems to be at least plausible. This implies that the supernova went off inside a low-density bubble close to the edge. Alternatively, if the plasma temperature $(k T=0.8 \mathrm{keV}$ represents that of shocked ambient ISM as the limited spectral analysis indicates, a naive Sedov model can be used to set an age $\gtrsim 15 \mathrm{kyr}$, or, taking a non-detection in X-ray of the outer, radiative shell $(k T \lesssim 0.16 \mathrm{keV})$, an even larger age limit of $\gtrsim 30 \mathrm{kyr}$ would be implied.

\subsection{Radio spectral index}

Using the flux density measurements from Table 2, we estimate a spectral index of $\alpha=-0.29 \pm 0.01$ across the remnant (including pulsar and PWN) (Fig. 8). The radio spectral index is estimated from the slope of a linear least-squares regression, in logarithmic space. From all our radio images it is clear that emission from the putative PWN dominates the radio emission (see Section 3.4).

The overall radio continuum spectral index $\alpha=-0.29 \pm 0.01$ of the remnant (Fig. 8) adds further support to our claim of the PWN nature of the emission. This value of $\alpha$ falls below that of 


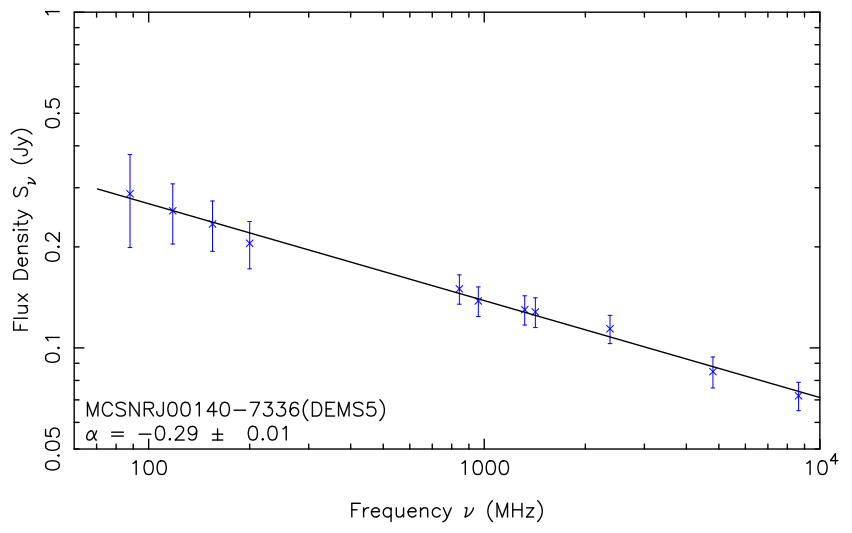

Figure 8. Radio continuum SED of PWN DEM S5.

a typical SNR of -0.5 (Bozzetto et al. 2017), and is in line with what is expected from an SNR/PWN system (e.g. the spectral index of the SNR and PWN for LMC SNR J0453-6829 is $-0.39 \pm 0.03$; Haberl et al. 2012b). As we were unable to disentangle the PWN emission from the rest of the shell, we were not able to estimate the spectral index of the SNR separately. We also note that the estimated value of $\alpha=-0.29$ could be somewhat flatter as our flux density estimates at lower frequencies includes the emission of the pulsar that might not be negligible but is a minor flux density contributor to the whole DEM S5 system (SNR-PWN-pulsar). We cannot rule out radio emission from the shell but the present radio images show little evidence of any, so we assume that all the radio emission is coming from the putative pulsar (compact source) and its PWN even though we recognize that some emission must come from the SNR itself.

\subsection{Search for the radio pulsar at Parkes}

On 2018 September 14 we observed the point-like source for $2700 \mathrm{~s}$ using the Directors Time of the ATNF Parkes radio telescope in NSW, Australia. The central beam of the multibeam receiver was used and data were recorded using the Parkes Digital Filterbank systems. We used a 2-bit sampling and a sampling time of $64 \mu \mathrm{s}$ with 512 frequency channels over $256 \mathrm{MHz}$ bandwidth (centred at $1369 \mathrm{MHz}$ ). The pulsar searching software package PRESTO (Ransom 2001) was used to carry out RFI zapping and masking, de-dispersion and searches of periodic signals. We searched for dispersion measures (DMs) of up to $1000 \mathrm{~cm}^{-3} \mathrm{pc}$, but found no convincing candidates with an $\mathrm{S} / \mathrm{N}$ greater than 8 . For a pulse period of $10 \mathrm{~ms}$ and DM of $200 \mathrm{~cm}^{-3} \mathrm{pc}$ and assuming a pulse duty cycle of 10 per cent, our observations provide a flux density limit of $0.24 \mathrm{mJy}$ at $1369 \mathrm{MHz}$ (see e.g. equation 9 of Dai, Johnston \& Hobbs 2017).

\subsection{Radio polarization}

Linear polarization images of DEM S5 at $5500 \mathrm{MHz}$ were created using the $Q$ and $U$ Stokes parameters as shown in Fig. 2. However, no reliable polarization images could be created at any other frequency, due to the poor signal-to-noise ratio $(9000 \mathrm{MHz})$ and depolarization (2100 MHz) and/or missing short spacings. The fractional polarization at $5500 \mathrm{MHz}$ has been evaluated using the standard MIRIAD task impol. The majority of the polarized emission is located at the position of the suggested PWN. Our estimated peak fractional polarization value is $P=32 \pm 7$ per cent while average polarization is about 23 percent. Such polarization that we detected in this area would disfavour extragalactic origin of the object and strongly advocate for the SMC birthplace. Moreover, this is in agreement with our other LMC PWN measurements such as Haberl et al. (2012b).

\subsection{Optical morphology}

The outer borders of DEM S5 show that [O III] emission dominates in this part of the remnant (Figs 6, 7, and 9). The X-rays show no oxygen enhancement and the [O III] emission clearly delineates the outer blast waves (Fig. 9; right-hand panel). This indicates that it is likely caused by radiative shocks, since [O III] is the strongest coolant of gas at a few $10^{5} \mathrm{~K}$. Since DEM S5 is probably in a radiative phase it presents very different characteristics than found in much younger SNR like Cas A or G292.0+1.8.

DEM 55 is some $300 \mathrm{pc}$ west of the main body of the SMC where the stellar environment and star formation would favour a Type Ia scenario (Maggi et al. 2016). This is the only known SMC SNR with a lack of recent star formation in the SFH map of Harris \& Zaritsky (2004). All other SMC SNRs are associated with a recent peak in $\mathrm{SFH}$, although some might be just in projection. However, it is possible that the progenitor of DEM S5 was itself a companion in a binary system that got ejected when the primary exploded (i.e. a runaway star), as the linear distance travelled by a runaway star would be $\sim 1000(t / 10 \mathrm{Myr})\left(v / 100 \mathrm{~km} \mathrm{~s}^{-1}\right) \mathrm{pc}$. Therefore, it is possible that if the progenitor of DEM S5 was in a binary system with a much more massive (and thus short-lived) companion, it had time to reach its current location, devoid of intense recent star-forming activity. Since the orientation of the PWN propagation is also away from the main body of the SMC, we consider the possibility that the motion of DEM S5 PWN could reflect the peculiar velocity of the progenitor star instead of a kick. However, the runaway velocity is unlikely to be that large, so possibly some compounded velocities (progenitor runaway + pulsar kick) are needed.

The central region is dominated by a keV-temperature thermal plasma from the X-rays (Fig. 9) that runs in the north-south direction of DEM S5. Diffuse X-ray emission to the left (east) in the interior is likely thermal emission from either shock-heated ISM or ejecta that was overran by the reverse shock, which now should have dissipated for the whole SNR. Therefore, the SNR is probably in a late stage of evolution and the missing or very weak radio synchrotron emission should support that as well as the missing thermal X-ray emission from the shell. The radius differences seem to indicate that the right-hand part (west) is further evolved and therefore expanding in a medium of higher density. The missing or very weak X-ray emission from the ejecta should support that too. We also recognize a somewhat smaller circular shell at the west side of DEM S5 in $\mathrm{H} \alpha$ and [S II] bands. This could be because of a density gradient across the entire SNR. If so, the explosion site might not be at the geometric centre but offset to the west which could result in a somewhat lower putative pulsar kick velocity.

\subsection{Infrared morphology}

We used the Spitzer SAGE survey (Gordon et al. 2011) to search for any extended mid-IR emission in the direction of DEM S5. We found enhanced emission in several bands with $8 \mu \mathrm{m}$ (IRAC band4) being the most prominent (see Fig. 10). The IR emission clearly extends towards the north-west of DEM S5 and tightly follows the $[\mathrm{S}$ II $] / \mathrm{H} \alpha$ emission in this region just above the radio PWN. Neither the optical nor the IR emission peaks precisely at the putative pulsar 


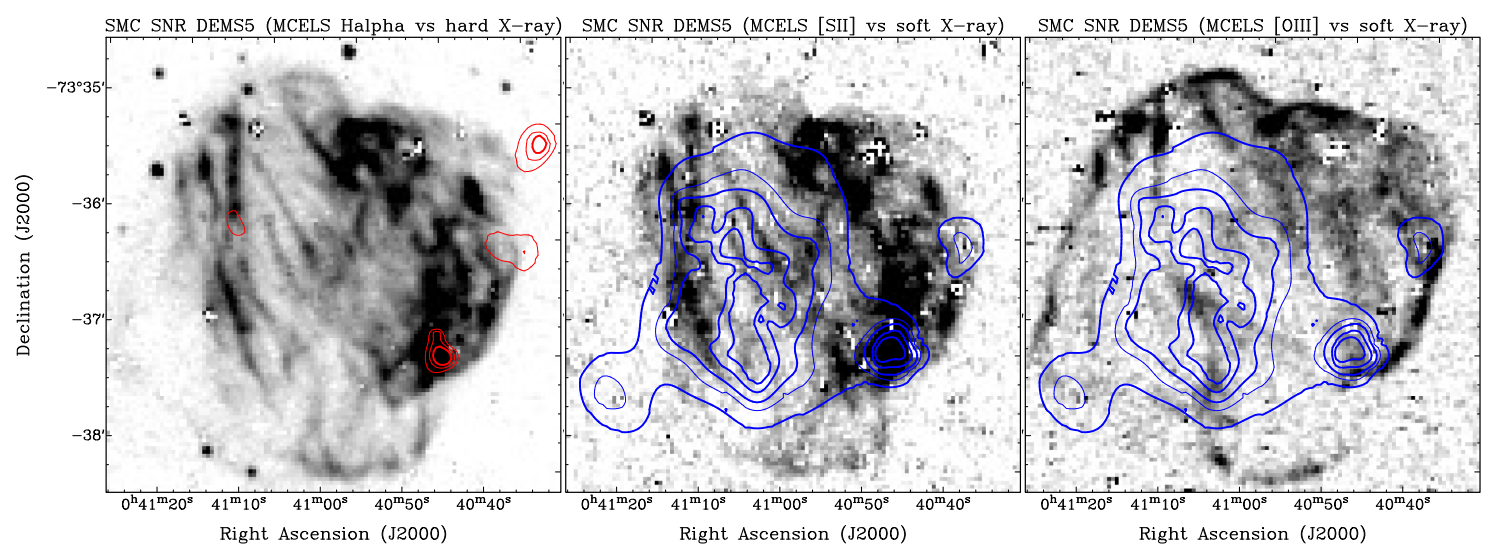

Figure 9. MCELS images of DEM S5 in $\mathrm{H} \alpha$ [left; overlaid with the hard X-ray (2-4.5 keV) contours (red)], [S II] and [O III] [middle and right-hand panels; overlaid with the soft band $(0.2-1 \mathrm{keV}) \mathrm{X}$-ray contours (blue)]. X-ray contour levels are the same as in Fig. 6.

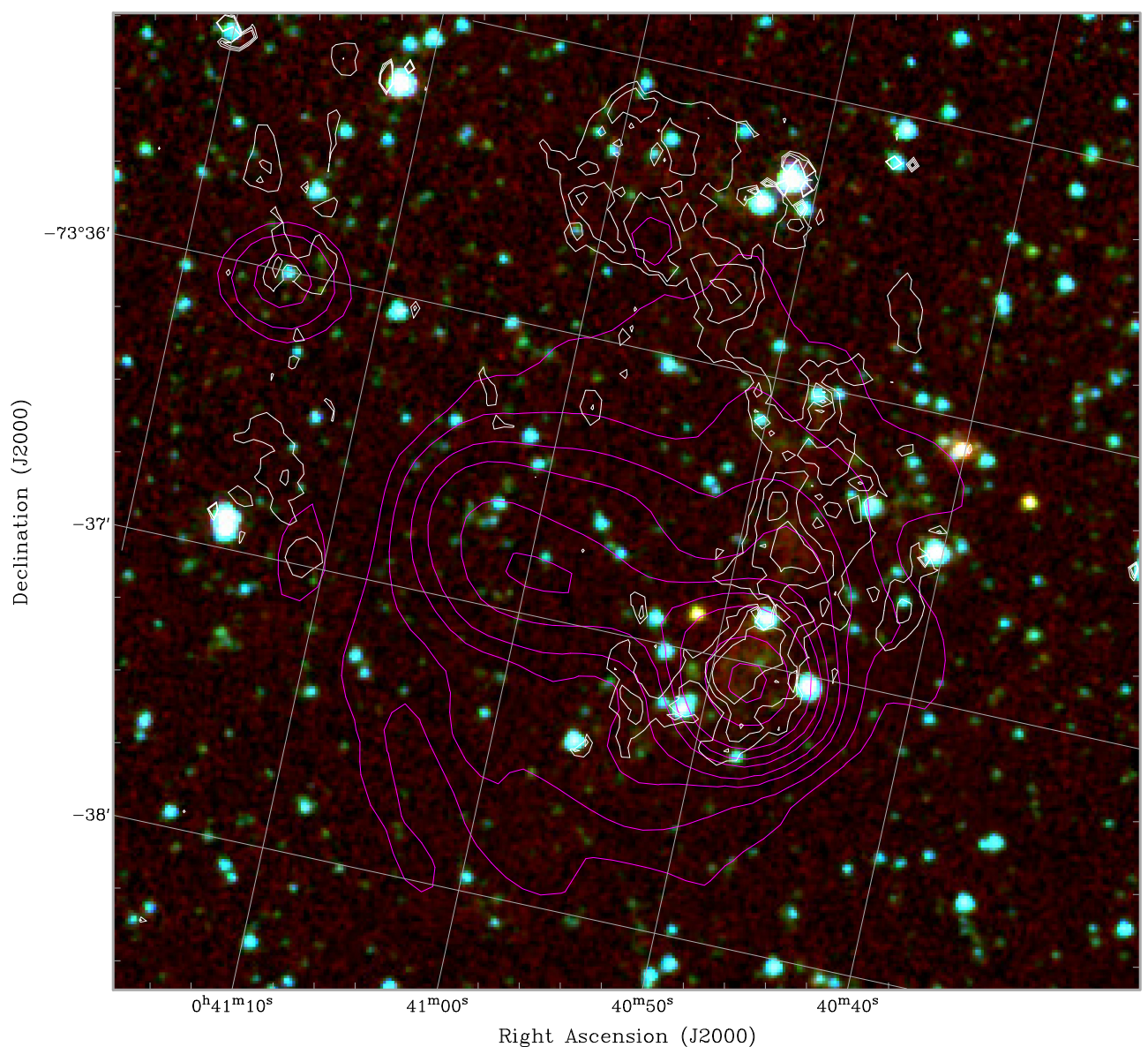

Figure 10. Spitzer RGB colour image of DEM S5 overlaid with ASKAP (1320 MHz) contours (magenta) and [S II] in white. Contours are the same as in Fig. 1. RGB images correspond to Spitzer IRAC band-4 (R; $8 \mu \mathrm{m}$ ), IRAC band-2 (G; $4.5 \mu \mathrm{m})$, and IRAC band-1 (B; $3.6 \mu \mathrm{m})$. Mid-IR emission nicely matches [S II] (and $\mathrm{H} \alpha$ ) indicating the presence of shocked gas.

position. However, we interpret this displacement as shocked gas trailing the whole PWN DEM S5 system. Alternatively, it could come from the SNR shock itself.

To ascertain the nature of the mid-IR nebulosity, we measure the integrated flux density in the brightest hotspots, and compare across the IRAC colours to discern the dominant emission components. The field is dense with stars, notably in the IRAC1 band image, and we carefully choose the apertures to avoid stars while also maximizing the diffuse emission captured in the aperture. Three positions are identified, with apertures chosen to be 7.5 arcsec in radius, centred on the equatorial J2000 positions: (\#1: $\left.10.203408^{\circ},-73.617633^{\circ}\right),\left(\# 2\right.$ : $\left.10.198245^{\circ},-73.608746^{\circ}\right)$, 
and (\#3: $\left.10.225005^{\circ},-73.594583^{\circ}\right)$. These positions correspond to the brightest IRAC-4 band nebulosity near the PWN and along the northern extension; see Figs 10 and 11.

Emission is detected in all four IRAC bands for the three positions; the integrated flux density measurements (magnitude and mJy units) for the first and brightest position (marked \#1 in Fig. 11) are shown in Table 6. We then compute the IRAC colours ratios: $F(4.5) / F(8.0)$ and $F(3.6) / F(5.8)$, which are used to decode the emission. For the brightest hotspot, the corresponding colours are 0.56 and 0.41 , respectively. The other two positions have similar colours: $(0.46,0.37)$ and $(0.44,0.35)$ for positions \#2 and \#3, respectively. The errors of the IRAC colour ratios are small ( $<$ few per cent). We now consider the nature of the emission based on these colours.

Mid-IR emission arises from atomic, molecular and dust species, excited by star formation and shocks in the ISM. The four broadbands of IRAC have been effectively used to study SNRs in the MW, including those embedded within dense gas. Reach et al. (2006) modelled the major emission mechanisms and created a set of diagnostics to assess the mid-IR emission associated with SNRs (see their figs 1 and 2, Reach et al. 2006). They considered thermal (star formation) and shock excitation, with the latter divided between the diffuse and dense gas $\left(n \sim>10^{2} \mathrm{~cm}^{-3}\right)$ ISM. For example, where ionic line emission dominates, the IRAC- 3 and four bands tend to be very bright relative to the other bands due to the presence of strong [Ar II] and [Fe II] lines (see their fig. 1). The Galactic SNR RCW103 is a classic example of IRAC colours influenced by ionic-dominated lines. Alternatively, if the blast wave and subsequent shock passes through dense gas, the dominant coolant is molecular hydrogen, and in conjunction with a molecular $\mathrm{CO}$ band head in the IRAC-2 band window, the colours conspire to look very different from the ionic case. The other Galactic SNR IC443 is an example of an SNR embedded within a molecular cloud. The colour-colour diagnostic of Reach et al. (2006) is shown in Fig. 12, where we have plotted the colours of the DEM S5 mid-IR nebulosity.

Based on the IRAC mid-IR emission diagnostic, the DEM S5 nebulosity is consistent with shock-excited molecular hydrogen. It has colours that are similar to the notably (molecular cloud) embedded SNRs, such as in Galactic SNRS IC443, W44, G311.5, and G346.6 (see fig. 22, Reach et al. 2006). The IR emission is spatially coincident with the radio continuum (see Fig. 11), which may indicate synchrotron contribute to the IR signal at some low level. Nevertheless, the IRAC colours clearly indicate that molecular emission is the dominant cooling mechanism of the shock front seen in DEM S5.

While it is not unusual to detect SNRs in the IR bands (see e.g. Reach et al. 2006; Bojičić et al. 2007; Lakićević et al. 2015) this would be the first bow shock supersonic PWN that potentially shows IR emission compared to a population of 28 described by Kargaltsev et al. (2017, see their table 1). However, there are a few cases in our Galaxy and in the LMC of detected IR emission from PWN, e.g. B0540-69.3 (Williams et al. 2008; Brantseg et al. 2014), G54.1+0.3 (Temim et al. 2010), and others. Still, none of these bright IR SNRs have bow shock PWN that moves supersonically. At the same time, the prominent IR emission from DEM S5 clearly following the $[\mathrm{S}$ II] emission which would therefore argue for a more classical SNR origin, with the IR emission associated with the outer SNR shock rather than with the PWN/bow shock. Also, DEM S5 is just the second SMC SNR (among a 25 SNR strong population) detected in IR along with SMC SNR J0106-7205 (a.k.a. IKT 25) (Koo et al. 2007) indicating that the SNR appearance in IR frequencies are indeed rare. However, the sensitivity of the present generation of IR telescopes and especially at the distance of the SMC might play an important role in the poor detection rate of IR emission from the SMC SNRs.

\subsection{H I morphology}

Fig. 13 shows the velocity channel maps of H I obtained with the ATCA and Parkes telescopes (Stanimirovic et al. 1999). We find two prominent Hi peaks at the radial velocities of $\sim 107 \mathrm{~km} \mathrm{~s}^{-1}$ (hereafter low-velocity cloud) and $\sim 166 \mathrm{~km} \mathrm{~s}^{-1}$ (hereafter highvelocity cloud). The intensity peak of the high-velocity cloud is $\sim 100$ pc away from the SNR (Fig. 13; bottom middle panel) and unlikely to be associated. For the low-velocity cloud the intensity peak shows a good spatial correspondence with the position of the SNR (Fig. 13; top middle panel), and a possible cavity-like structure is seen (top left panel). Recent data taken with the ASKAP telescope of the SMC (McClure-Griffiths et al. 2018) shows this cavity in more detail in Fig. 14. The ASKAP data has three times better spatial resolution $(35 \times 27 \mathrm{arcsec})$ compared to ATCA data and an rms of $0.7 \mathrm{~K}$ per a $3.9 \mathrm{~km} \mathrm{~s}^{-1}$ wide spectral channel. Fig. 14 shows a filament like H I structure that has a cavity corresponding to the location and size of the $\mathrm{H} \alpha$ emission of SNR DEM S5. A moment map in this velocity range of $96-104 \mathrm{~km} \mathrm{~s}^{-1}$ of the ASKAP data is shown in Fig. 15.

However, the radius of this cavity $(\sim 70 \mathrm{pc})$ is over twice as large as the SNR, so may not be directly associated, or may represent the combined energy input from the progenitor and previous generations of nearby stars and SNe. Possible line-splitting is present. If interpreted as shell expansion, the expansion velocity is $\sim 8 \mathrm{~km} \mathrm{~s}^{-1}$, which leads to a dynamical age of $5 \mathrm{Myr}$, an average mechanical luminosity of $\sim 400 \mathrm{~L}_{\odot}$ and a total energy of $2 \times 10^{50} \mathrm{erg}$, assuming an ambient density of $2 \mathrm{~cm}^{-3}$ (Staveley-Smith et al. 1997). These values indicate that an association with the SNR is plausible, particularly if the SNR has re-accelerated a shell or pre-existing structure created by past events.

\section{DISCUSSION}

We explore two possible scenarios for the nature of this object. Namely:

(i) A PWN associated with DEM S5: This is supported by (a) the detection of a radio point source coincident with the hard Xray point source (PWN/pulsar composite), (b) an X-ray spectrum consistent with a power law with photon index typical as seen from PWN/pulsar spectra and the stability of the X-ray flux, (c) the radio spectral indices and (d) shocked IR emission. In the case of PWNe, the extent of the radio emission is generally greater than that in X-rays because of the longer cooling time of radioemitting electrons. The radio emission may therefore trace the path of the PWN from the centre of the SNR as indicated by the current radio data of DEM S5 at $2100 \mathrm{MHz}$ (Fig. 6). The detection of the cometary morphology in radio indicates a large kick velocity of the pulsar at birth. The estimated distance between the point-like source and the centre of the SNR indicates that the transverse velocity of the putative pulsar could be as high as $\sim 2000 \mathrm{~km} \mathrm{~s}^{-1}$ with an age of 10-15 kyr. The lower and somewhat more realistic limits would put this SNR/PWN system into $\sim 28 \mathrm{kyr}$ age and kick-off velocity of $700-800 \mathrm{~km} \mathrm{~s}^{-1}$. Determination of the putative pulsar velocity is important to determine the birth kick distribution of the NS that can further constrain supernova explosion models and kick mechanisms (e.g. Ng \& Romani 2007). Finding such a source in 

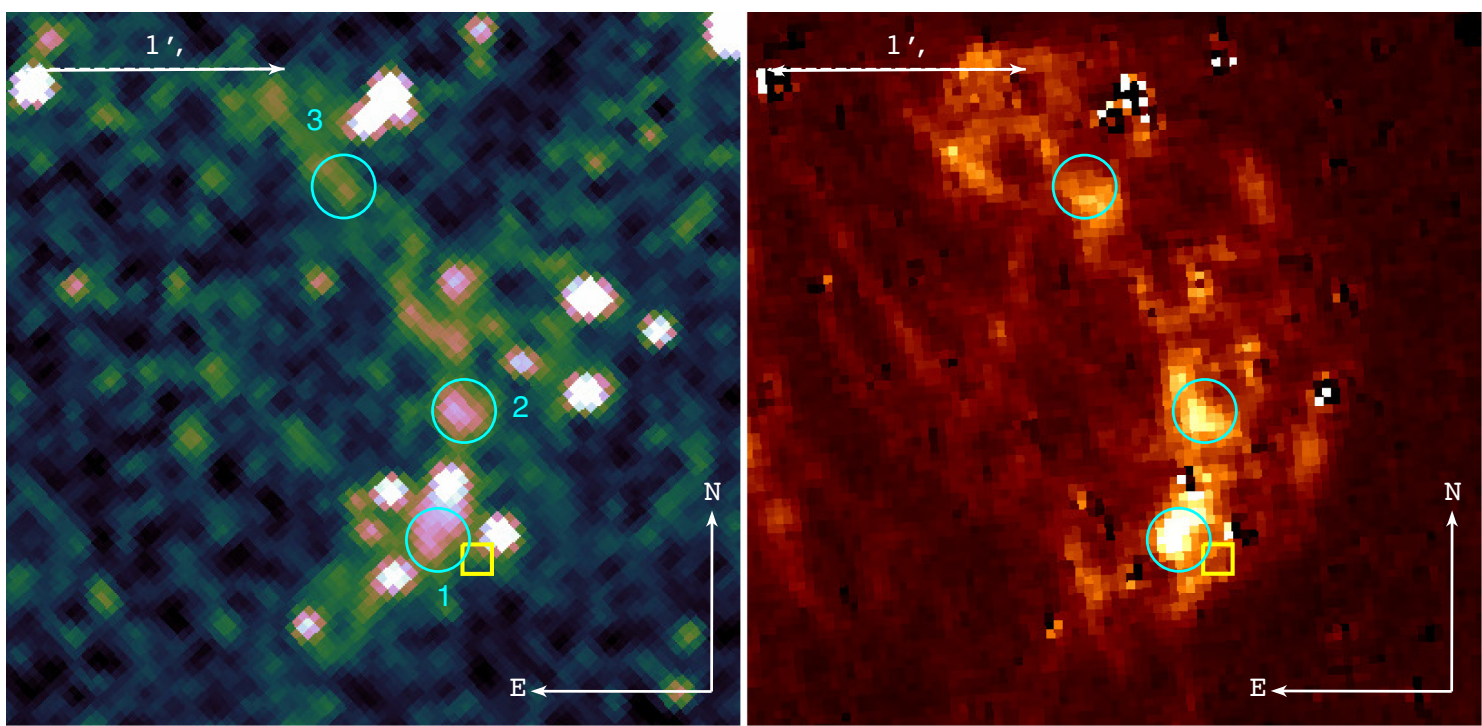

Figure 11. Spitzer western shock front of SNR DEM S5 as viewed in the IRAC-4 band ( $8 \mu \mathrm{m}$; left-hand panel) and the MCELS [S II] (right-hand panel). Approximate position of the pulsar is marked with the yellow box. Indicated are the three apertures used to measure the mid-IR emission, each is 7.5 arcsec in radius, centred on the bright emission and avoiding field stars. These mid-IR hotspots are also bright in the radio continuum, indicating that at least some (if not most) of the emission arises from synchrotron.

Table 6. Integrated flux density ( $7.5 \mathrm{arcsec}$ radius aperture) of the mid-IR emission for the brightest hotspot (\#1 from Fig. 11).

\begin{tabular}{lcccccc}
\hline Band & $\begin{array}{c}\text { RA(J2000) } \\
(\mathrm{deg})\end{array}$ & $\begin{array}{c}\text { Dec.(J2000) } \\
(\mathrm{deg})\end{array}$ & $f(\mathrm{mJy})$ & $\mathrm{mag}$ & $\mathrm{dmag}$ & $\mathrm{S} / \mathrm{N}$ \\
\hline IRAC-1 & 10.20294 & -73.61740 & 0.146 & 15.714 & 0.396 & 2.7 \\
IRAC-2 & 10.20309 & -73.61750 & 0.236 & 14.704 & 0.309 & 3.5 \\
IRAC-3 & 10.20324 & -73.61760 & 0.357 & 13.769 & 0.272 & 4.0 \\
IRAC-4 & 10.20380 & -73.61760 & 0.425 & 12.946 & 0.236 & 4.6 \\
\hline
\end{tabular}

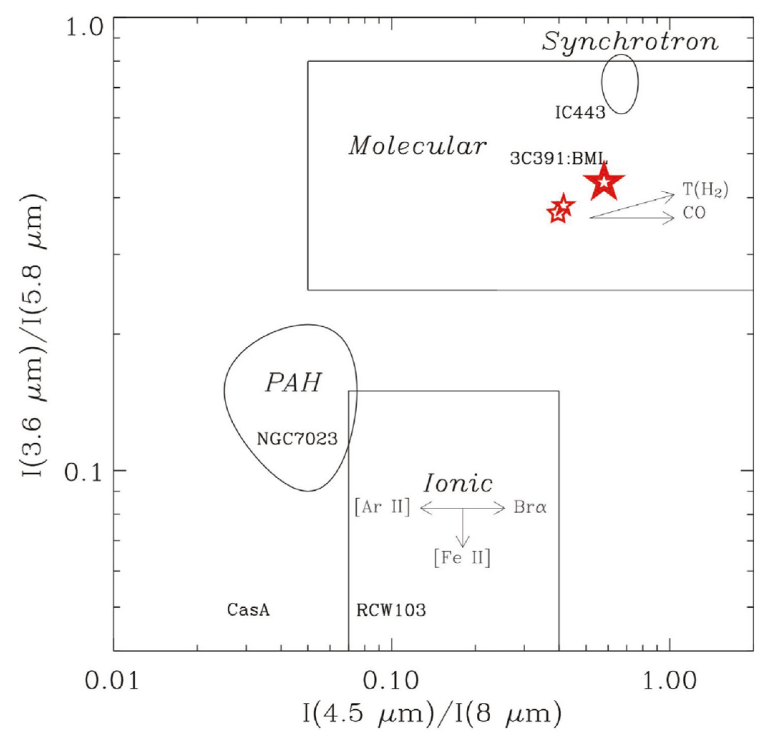

Figure 12. Spitzer IRAC colour-colour diagram, adapted from Reach et al. (2006), shows where the dominant emission mechanism appear in colour space. Three basic classes are shown: $\mathrm{PAH}$ and $\mathrm{HII}$ emission from star formation, ionic shock emission, and molecular shock emission. Based on the measurements in the three apertures (Figs 10 and 11), the midIR emission in DEMS5 has colours (indicated by the red stars) that clearly indicate molecular hydrogen emission is dominating the broad-band imaging. the SMC is particularly exciting as there might be differences in the pulsar birth, explosion and kick properties in the low-metallicity environment of SMC.

To some extent DEMS5 resembles similar morphological appearance like two well-studied LMC SNRs - MCSNR J05086902 (Bozzetto et al. 2014) and MCSNR J0527-7104 (Kavanagh et al. 2016) as well as, for example, Galactic SNR MSH 11-61A (Filipović, Payne \& Jones 2005a) or G332.5-5.6 (Stupar et al. 2007). While two LMC SNRs are classified as a likely Type Ia, they all have apparent 'mixed-morphology' appearance where SNR looks quite different at different wavebands. Mixed-morphology SNRs have shell-like radio morphologies combined with centre-filled Xray morphologies. The central X-ray morphology is produced by thermal emission rather than a central PWN (Rho \& Petre 1998; Pannuti et al. 2017). What makes DEM S5 different from other so-called mixed-morphology SNRs is its bow shock PWN.

(ii) An AGN in the background of the SMC: It is possible that the hard X-ray point source is not associated with the SNR but is instead a background AGN coincident with the SMC. The diffuse radio emission would in this case be due to a radio lobe or jets. A greater absorption component would then be expected to account for the line of sight through the SMC, although this may be sometimes balanced by a soft excess component (Turner \& Pounds 1989). As seen in Section 2.2, the absorption at the source position through the depth of the SMC indicates that the source is most likely located in the SMC. We also searched for a possible AGN association of the source using the ALLWISE criterion in the mid-IR and from the existing quasar catalogues. We did not find any AGN within $3 \sigma$ of the source position (Maitra et al. 2018).

\section{CONCLUSIONS AND FUTURE STUDIES}

We have presented results of a newly discovered pulsar-powered nebula associated with SNR DEMS5 in the SMC. The object exhibits a cometary morphology that suggests a pulsar leading the PWN and travelling supersonically through the ambient medium. This is the first detected extragalactic source to exhibit 

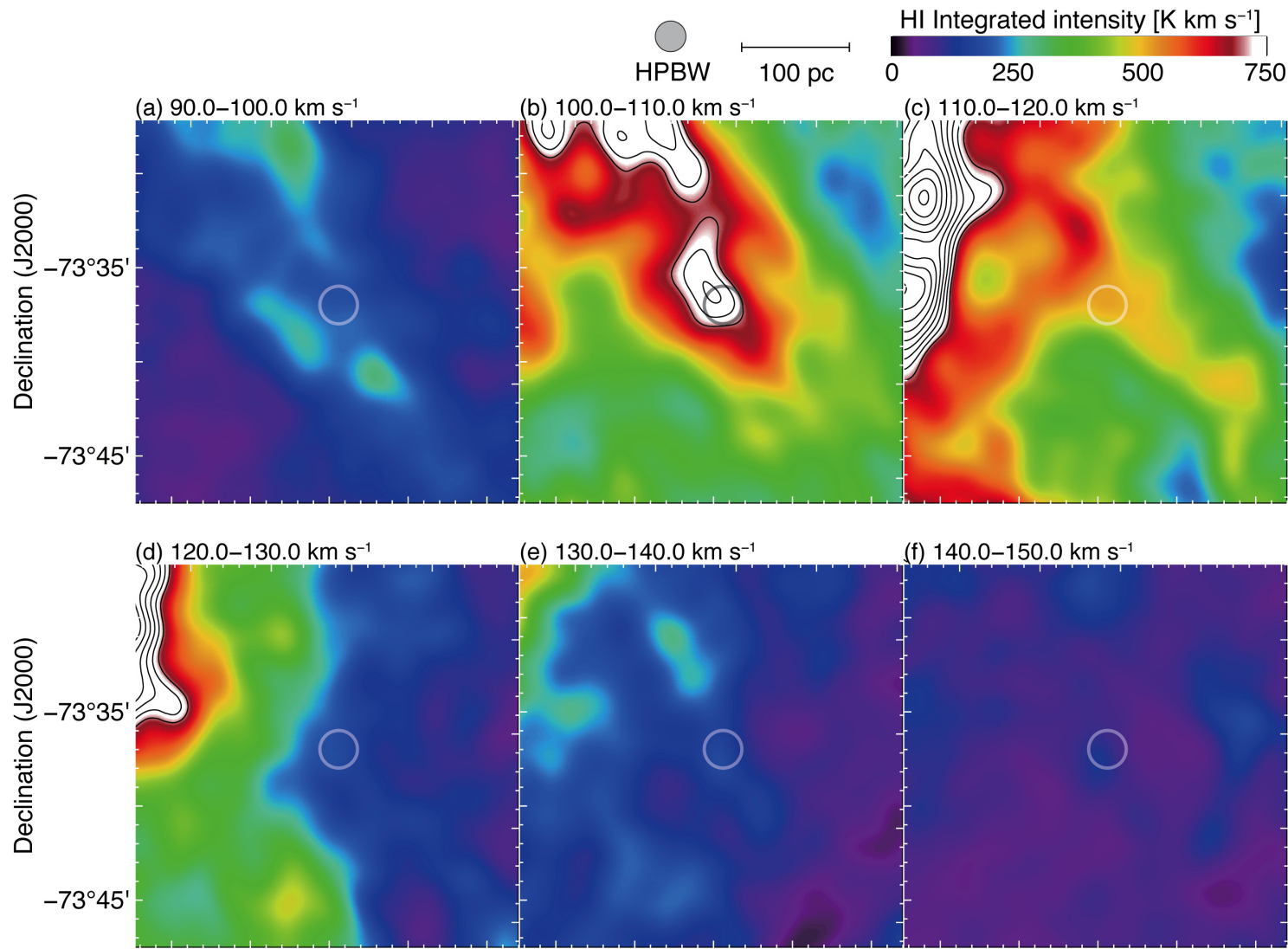

(e) $130.0-140.0 \mathrm{~km} \mathrm{~s}^{-1}$

(f) $140.0-150.0 \mathrm{~km} \mathrm{~s}^{-1}$

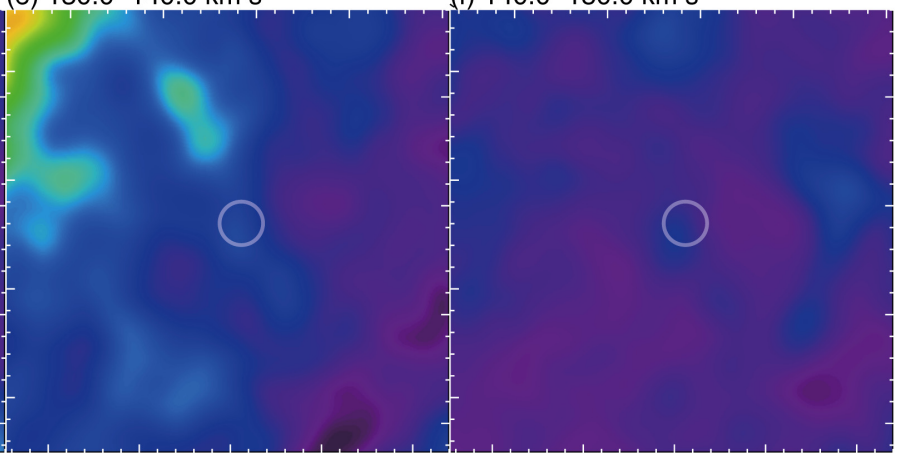

(g) $150.0-160.0 \mathrm{~km} \mathrm{~s}^{-1}$

(h) $160.0-170.0 \mathrm{~km} \mathrm{~s}^{-1}$

(i) $170.0-180.0 \mathrm{~km} \mathrm{~s}^{-1}$

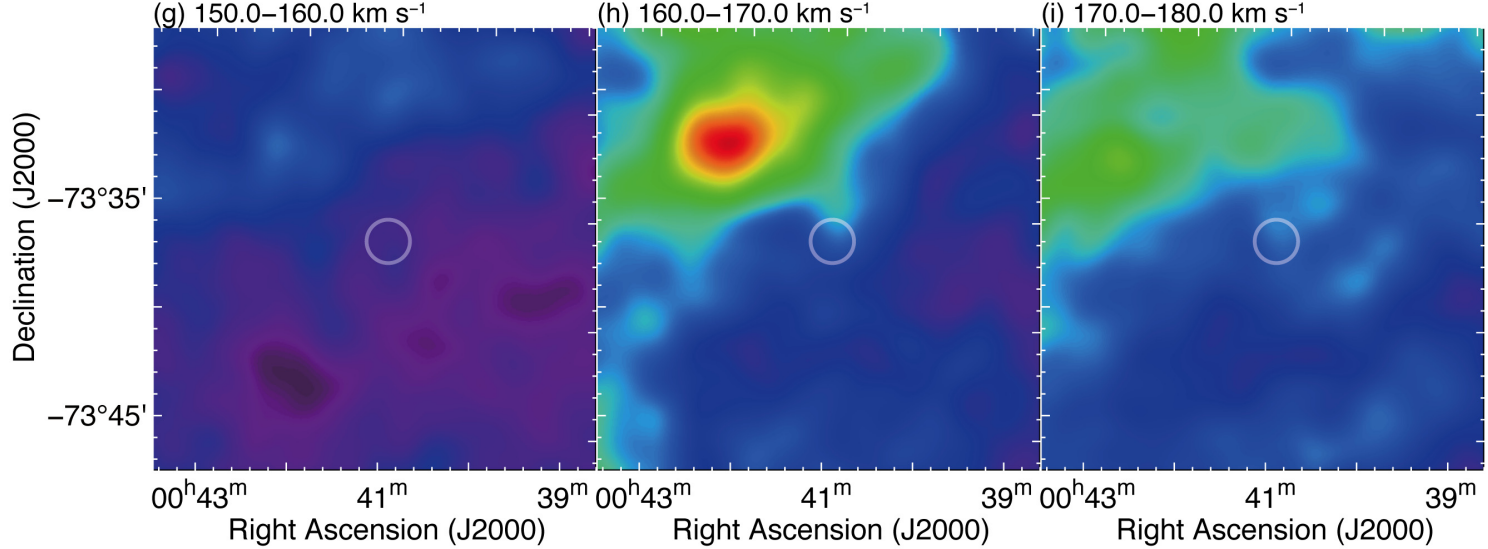

Figure 13. Velocity channel maps of H I towards SNR DEM S5 obtained with the ATCA and Parkes telescopes (Stanimirovic et al. 1999). Each panel shows $\mathrm{HI}$ intensity distribution integrated every $10 \mathrm{~km} \mathrm{~s}^{-1}$ in a velocity range from 90 to $180 \mathrm{~km} \mathrm{~s}^{-1}$. Superposed circles indicate a shell boundary of the SNR. The scale bar and beam size of $\mathrm{HI}$ are also shown.

this type of morphology. The distance of this object $(\sim 21.2 \mathrm{pc})$ from the centre of DEMS5 SNR would require a kick velocity as high as $\sim 2000 \mathrm{~km} \mathrm{~s}^{-1}$ and as low as $\sim 700 \mathrm{~km} \mathrm{~s}^{-1}$ for the pulsar at birth. The SNR/PWN/pulsar system exhibits a typically flat SED of $-0.29 \pm 0.01$ inline with most other PWNe. We found no convincing DMs candidates (up to $1000 \mathrm{~cm}^{-3} \mathrm{pc}$ ) with an $\mathrm{S} / \mathrm{N}$ greater than 8 . We also detect radio polarization in the locale of the emission from this object with a peak (fractional) value at $5500 \mathrm{MHz}$ of $P=32 \pm 7$ percent and average of $\sim 23$ per cent that strongly indicates non-thermal emission. At IR frequencies, we detect associated shock emission that indicates that the DEM S5 SNR is still expanding. Finally, our H I velocity channel maps show possible interaction with the low-velocity cloud at $\sim 107 \mathrm{~km} \mathrm{~s}^{-1}$.

Future follow-up multifrequency observations of this object with a high spatial resolution in X-rays will be able to accurately measure the extent of the hard X-ray point source within DEMS5, and resolve the compact object from the diffuse nebula. We will also search for a radio pulsar at this position. Another objective will be to accurately measure the position of the compact object within DEM S5, which will help to better constrain the motion of the pulsar within the SNR and identify its counterpart at other wavelengths.

Finally, the study of PWNe at TeV gamma-ray energies (where the inverse-Compton process provides the gamma-ray photons) 

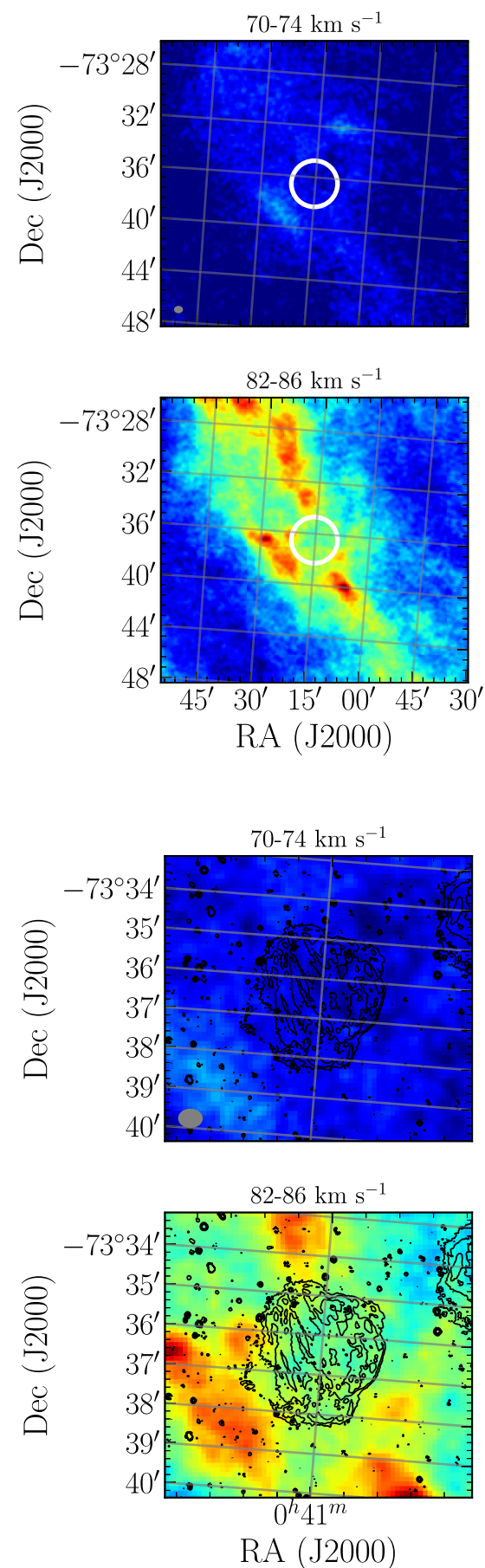

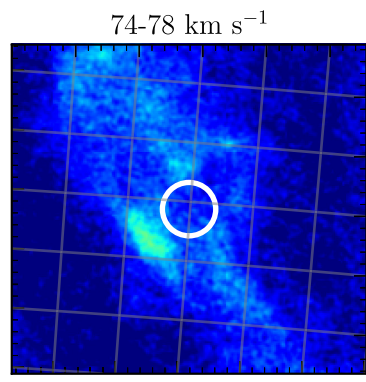

$86-90 \mathrm{~km} \mathrm{~s}^{-1}$

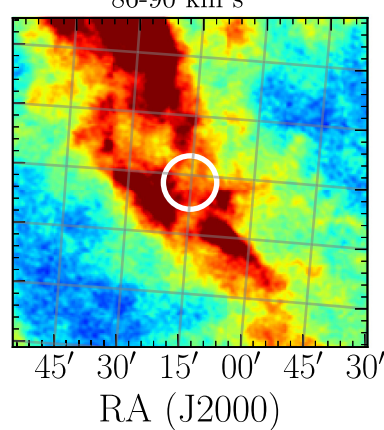

$74-78 \mathrm{~km} \mathrm{~s}^{-1}$

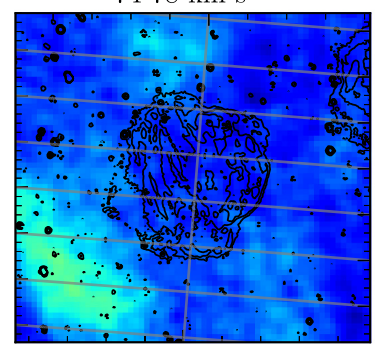

$86-90 \mathrm{~km} \mathrm{~s}^{-1}$

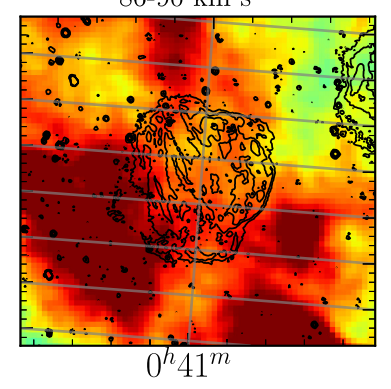

RA (J2000)

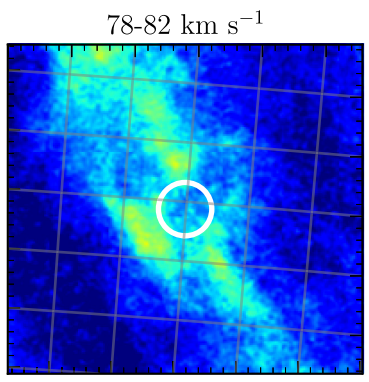

$90-94 \mathrm{~km} \mathrm{~s}^{-1}$
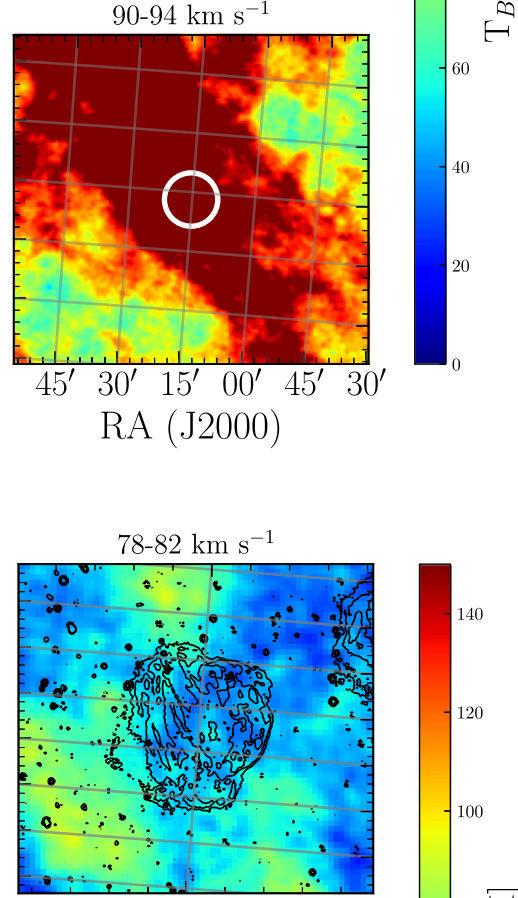

$90-94 \mathrm{~km} \mathrm{~s}^{-1}$

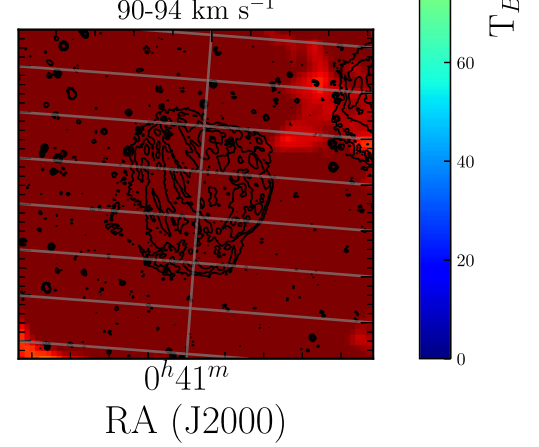

Figure 14. Integrated H moment maps (70-94 $\mathrm{km} \mathrm{s}^{-1}$ ) towards SNR DEM S5 obtained with the ASKAP and Parkes telescopes (McClure-Griffiths et al. 2018). The maps are integrated over $4 \mathrm{~km} \mathrm{~s}^{-1}$. A colour bar is shown on the right-hand side and the beam size is shown at the bottom left corner of the first images. The top panel shows a 22 arcmin region around SNR DEM S5. The white circle indicates the size and location of the SNR. The bottom panel shows the same region zoomed into the inner 7 arcmin and with the $\mathrm{H} \alpha$ contours $\left(25,50,100\right.$, and $\left.200 \times 10^{-17} \mathrm{ergs} \mathrm{cm}^{-2} \mathrm{~s}^{-1}\right) \mathrm{overlayed}$

allows important constraints on the magnetic field and transport properties of the PWN electrons (e.g. Aharonian, Atoyan \& Kifune 1997). The now well-established X-ray and TeV gamma-ray emission correlation in PWNe (Mattana et al. 2009; Kargaltsev, Rangelov \& Pavlov 2013; H. E. S. S. Collaboration 2018) can provide a prediction of the potential $\mathrm{TeV}$ flux. For the pulsar spin-down power and characteristic age of DEMS5, the TeV flux could be a factor 5 to 10 times higher than its X-ray flux $\left(3.6 \times 10^{-14} \mathrm{erg} \mathrm{cm}^{-2} \mathrm{~s}^{-1}\right)$. Such a TeV flux level is within the reach of the forthcoming Čerenkov Telescope Array (CTA; Martin et al. 2019; Cherenkov Telescope Array Consortium et al. 2019). A similar X-ray to TeV flux scaling may apply to the other PWN in the SMC, IKT 16 (Maitra et al. 2015), bringing it within the reach of the CTA. 


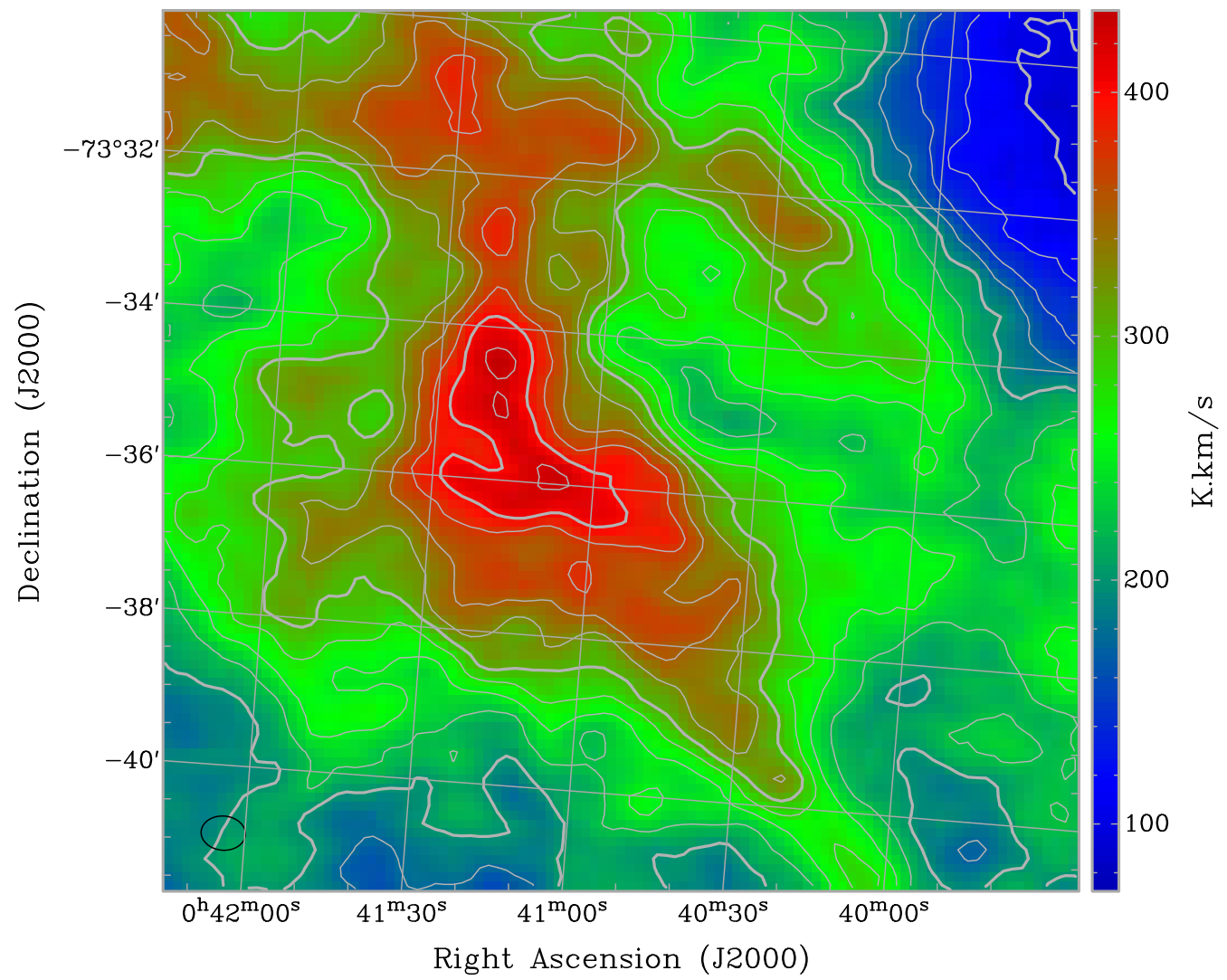

Figure 15. An integrated H I moment map towards SNR DEM S5 obtained with the ASKAP and Parkes telescopes (McClure-Griffiths et al. 2018). The map is integrated over two channels $\left(8 \mathrm{~km} \mathrm{~s}^{-1}\right)$ centred at a heliocentric velocity of $100 \mathrm{~km} \mathrm{~s}^{-1}$. The contours are spaced at intervals of $25 \mathrm{~K} \mathrm{~km} \mathrm{~s}^{-1}$ starting at $75 \mathrm{~K} \mathrm{~km} \mathrm{~s}^{-1}$. Bold contours are at intervals of $100 \mathrm{~K} \mathrm{~km} \mathrm{~s}^{-1}$ starting at $100 \mathrm{~K} \mathrm{~km} \mathrm{~s}^{-1}$. A colour bar is shown on the right-hand side and the beam size is shown at the bottom left.

\section{ACKNOWLEDGEMENTS}

The Australian Compact Array and the Australian SKA Pathfinder (ASKAP) are part of the Australian Telescope that is funded by the Commonwealth of Australia for operation as National Facility managed by CSIRO. This paper includes archived data obtained through the Australia Telescope Online Archive (http://atoa.atnf.csiro.au). We used the KARMA and MIRIAD software packages developed by the ATNF. Operation of ASKAP is funded by the Australian Government with support from the National Collaborative Research Infrastructure Strategy. ASKAP uses the resources of the Pawsey Supercomputing Centre. Establishment of ASKAP, the Murchison Radio-astronomy Observatory and the Pawsey Supercomputing Centre are initiatives of the Australian Government, with support from the Government of Western Australia and the Science and Industry Endowment Fund. We acknowledge the Wajarri Yamatji people as the traditional owners of the Observatory site. MS acknowledges support by the Deutsche Forschungsgemeinschaft (DFG) through the Heisenberg research grant SA 2131/4-1 and the Heisenberg professor grant SA 2131/5-1. This work is part of project No. 176005, 'Emission nebulae: structure and evolution', supported by the Ministry of Education, Science, and Technological Development of the Republic of Serbia. Parts of this research were conducted with support of Australian Research Council Centre of Excellence for All Sky Astrophysics in 3 Dimensions (ASTRO 3D), through project number CE170100013. The authors would like to thank the anonymous referee for a constructive report and useful comments.

\section{REFERENCES}

Aharonian F. A., Atoyan A. M., Kifune T., 1997, MNRAS, 291, 162 Arnaud K. A., 1996, in Jacoby G. H., Barnes J., eds, ASP Conf. Ser. Vol. 101, Astronomical Data Analysis Software and Systems V. Astron. Soc. Pac., San Francisco, p. 17

Barkov M. V., Lyutikov M., Khangulyan D., 2019, MNRAS, 484, 4760

Besla G., Kallivayalil N., Hernquist L., Robertson B., Cox T. J., van der Marel R. P., Alcock C., 2007, ApJ, 668, 949

Bojičić I. S., Filipović M. D., Parker Q. A., Payne J. L., Jones P. A., Reid W., Kawamura A., Fukui Y., 2007, MNRAS, 378, 1237

Bozzetto L. M. et al., 2012, MNRAS, 420, 2588

Bozzetto L. M. et al., 2014, MNRAS, 439, 1110

Bozzetto L. M. et al., 2017, ApJS, 230, 2

Brantseg T., McEntaffer R. L., Bozzetto L. M., Filipovic M., Grieves N., 2014, ApJ, 780, 50

Cherenkov Telescope Array Consortium, 2019, Science with the Cherenkov Telescope Array. World Scientific, Singapore

Cioffi D. F., McKee C. F., Bertschinger E., 1988, ApJ, 334, 252

Collier J. D., Norris R. P., Filipović M. D., Tothill N. F. H., 2016, Astron. Nachr., 337, 36

Crawford E. J., Filipovic M. D., de Horta A. Y., Wong G. F., Tothill N. F. H., Draskovic D., Collier J. D., Galvin T. J., 2011, Serb. Astron. J., 183, 95 
Crawford E. J., Filipović M. D., McEntaffer R. L., Brantseg T., Heitritter K., Roper Q., Haberl F., Urošević D., 2014, AJ, 148, 99

Dai S., Johnston S., Hobbs G., 2017, MNRAS, 472, 1458

Filipović M. D., Jones P. A., White G. L., Haynes R. F., Klein U., Wielebinski R., 1997, A\&AS, 121, 321

Filipović M. D., Haynes R. F., White G. L., Jones P. A., 1998, A\&AS, 130, 421

Filipović M. D., Bohlsen T., Reid W., Staveley-Smith L., Jones P. A., Nohejl K., Goldstein G., 2002, MNRAS, 335, 1085

Filipović M. D., Payne J. L., Jones P. A., 2005a, Serb. Astron. J., 170, 47

Filipović M. D., Payne J. L., Reid W., Danforth C. W., Staveley-Smith L., Jones P. A., White G. L., 2005b, MNRAS, 364, 217

Filipović M. D. et al., 2008, A\&A, 485, 63

For B.-Q. et al., 2018, MNRAS, 480, 2743

Gaensler B. M., van der Swaluw E., Camilo F., Kaspi V. M., Baganoff F. K., Yusef-Zadeh F., Manchester R. N., 2004, ApJ, 616, 383

Gooch R., 1995, in Shaw R. A., Payne H. E., Hayes J. J. E., eds, ASP Conf. Ser. Vol. 77, Astronomical Data Analysis Software and Systems IV. Astron. Soc. Pac., San Francisco, p. 144

Gordon K. D. et al., 2011, AJ, 142, 102

Haberl F., Sturm R., 2016, A\&A, 586, A81

Haberl F., Sturm R., Filipović M. D., Pietsch W., Crawford E. J., 2012a, A\&A, 537, L1

Haberl F. et al., 2012b, A\&A, 543, A154

Haberl F. et al., 2012c, A\&A, 545, A128

Han J., Wang C., Xu J., Han J.-L., 2016, Res. Astron. Astrophys., 16, 159

Hancock P. J., Murphy T., Gaensler B. M., Hopkins A., Curran J. R., 2012, MNRAS, 422, 1812

Hancock P. J., Trott C. M., Hurley-Walker N., 2018, PASA, 35, e011

Harris J., Zaritsky D., 2004, AJ, 127, 1531

H. E. S. S. Collaboration, 2018, A\&A, 612, A3

Holland-Ashford T., Lopez L. A., Auchettl K., Temim T., Ramirez-Ruiz E., 2017, ApJ, 844, 84

Hurley-Walker N. et al., 2017, MNRAS, 464, 1146

Kargaltsev O., Pavlov G. G., 2008, in Bassa C., Wang Z., Cumming A., Kaspi V. M., eds, AIP Conf. Ser. Vol. 983, 40 Years of Pulsars: Millisecond Pulsars, Magnetars and More. Am. Inst. Phys., New York, p. 171

Kargaltsev O., Rangelov B., Pavlov G. G., 2013, preprint (arXiv:1305.2552)

Kargaltsev O., Pavlov G. G., Klingler N., Rangelov B., 2017, J. Plasma Phys., 83, 635830501

Katsuda S. et al., 2018, ApJ, 856, 18

Kavanagh P. J. et al., 2016, A\&A, 586, A4

Klinger R. J., Dickel J. R., Fields B. D., Milne D. K., 2002, AJ, 124, 2135

Klingler N., Kargaltsev O., Pavlov G. G., Ng C.-Y., Beniamini P., Volkov I., 2018, ApJ, 861, 5

Koo B.-C. et al., 2007, PASJ, 59, S455

Kothes R., 2017, in Astrophysics and Space Science Library, Vol. 446, Radio Properties of Pulsar Wind Nebulae. Springer International Publishing, Cham, p. 1

Lakićević M. et al., 2015, ApJ, 799, 50

Macri L. M., Stanek K. Z., Bersier D., Greenhill L. J., Reid M. J., 2006, ApJ, 652, 1133

Maggi P. et al., 2016, A\&A, 585, A162

Maitra C., Ballet J., Filipović M. D., Haberl F., Tiengo A., Grieve K., Roper Q., 2015, A\&A, 584, A41

Maitra C., Ballet J., Esposito P., Haberl F., Tiengo A., Filipović M. D., Acero F., 2018, A\&A, 612, A87

Marelli M. et al., 2013, ApJ, 765, 36

Martin P., Lu C.-C., Voelk H., Renaud M., Filipovic M., 2019, 7: KSP: Large Magellanic Cloud Survey. World Scientific, Singapore, p. 125

Mattana F. et al., 2009, ApJ, 694, 12

McClure-Griffiths N. M. et al., 2018, Nat. Astron., 2, 901

McKee C. F., Truelove J. K., 1995, Phys. Rep., 256, 157

Moon D. S. et al., 2004, ApJ, 610, L33

Ng C.-Y., Romani R. W., 2007, ApJ, 660, 1357

Owen R. A. et al., 2011, A\&A, 530, A132

Pannuti T. G. et al., 2017, ApJ, 839, 59

Pavan L. et al., 2014a, Int. J. Mod. Phys. Conf. Ser., 28, 1460172
Pavan L. et al., 2014b, A\&A, 562, A122

Payne J. L., Filipović M. D., Reid W., Jones P. A., Staveley-Smith L., White G. L., 2004, MNRAS, 355, 44

Payne J. L., White G. L., Filipović M. D., Pannuti T. G., 2007, MNRAS, 376, 1793

Pellegrini E. W., Oey M. S., Winkler P. F., Points S. D., Smith R. C., Jaskot A. E., Zastrow J., 2012, ApJ, 755, 40

Ransom S. M., 2001, American Astronomical Society Meeting Abstracts, p. 119.03

Reach W. T. et al., 2006, AJ, 131, 1479

Reid W. A., Payne J. L., Filipović M. D., Danforth C. W., Jones P. A., White G. L., Staveley-Smith L., 2006, MNRAS, 367, 1379

Reynolds S. P., Pavlov G. G., Kargaltsev O., Klingler N., Renaud M., Mereghetti S., 2017, Space Sci. Rev., 207, 175

Rho J., Petre R., 1998, ApJ, 503, L167

Roper Q., McEntaffer R. L., DeRoo C., Filipovic M., Wong G. F., Crawford E. J., 2015, ApJ, 803, 106

Russell S. C., Dopita M. A., 1992, ApJ, 384, 508

Safi-Harb S., Ogelman H., Finley J. P., 1995, ApJ, 439, 722

Sault R. J., Wieringa M. H., 1994, A\&AS, 108, 585

Sault R. J., Teuben P. J., Wright M. C. H., 1995, in Shaw R. A., Payne H. E., Hayes J. J. E., eds, ASP Conf. Ser. Vol. 77, Astronomical Data Analysis Software and Systems IV. Astron. Soc. Pac., San Francisco, p. 433

Scowcroft V., Freedman W. L., Madore B. F., Monson A., Persson S. E., Rich J., Seibert M., Rigby J. R., 2016, ApJ, 816, 49

Smith C., Leiton R., Pizarro S., 2000, in Alloin D., Olsen K., Galaz G., eds, ASP Conf. Ser. Vol. 221, Stars, Gas and Dust in Galaxies: Exploring the Links. Astron. Soc. Pac., San Francisco, p. 83

Stanimirovic S., Staveley-Smith L., Dickey J. M., Sault R. J., Snowden S. L., 1999, MNRAS, 302, 417

Staveley-Smith L., Sault R. J., Hatzidimitriou D., Kesteven M. J., McConnell D., 1997, MNRAS, 289, 225

Strüder L. et al., 2001, A\&A, 365, L18

Stupar M., Parker Q. A., Filipović M. D., Frew D. J., Bojičić I., Aschenbach B., 2007, MNRAS, 381, 377

Sturm R. et al., 2013, A\&A, 558, A3

Temim T., Slane P., Reynolds S. P., Raymond J. C., Borkowski K. J., 2010, ApJ, 710, 309

Tiengo A., Esposito P., Mereghetti S., 2008, ApJ, 680, L133

Turner T. J., Pounds K. A., 1989, MNRAS, 240, 833

Turner M. J. L. et al., 2001, A\&A, 365, L27

Turtle A. J., Ye T., Amy S. W., Nicholls J., 1998, PASA, 15, 280

Weaver R., McCray R., Castor J., Shapiro P., Moore R., 1977, ApJ, 218, 377

Williams B. J. et al., 2008, ApJ, 687, 1054

Wong G. F., Filipovic M. D., Crawford E. J., de Horta A. Y., Galvin T., Draskovic D., Payne J. L., 2011a, Serb. Astron. J., 182, 43

Wong G. F. et al., 2011b, Serb. Astron. J., 183, 103

Wong G. F. et al., 2012a, Serb. Astron. J., 184, 93

Wong G. F., Filipovic M. D., Crawford E. J., Tothill N. F. H., De Horta A. Y., Galvin T. J., 2012b, Serb. Astron. J., 185, 53

${ }^{1}$ Western Sydney University, Locked Bag 1797, Penrith South DC, NSW 1797, Australia

${ }^{2}$ Max-Planck-Institut für extraterrestrische Physik, Giessenbachstraße, D85748 Garching, Germany

${ }^{3}$ Observatoire Astronomique de Strasbourg, Université de Strasbourg, CNRS, 11 rue de l'Université, F-67000 Strasbourg, France

${ }^{4}$ Remeis Observatory and ECAP, Universität Erlangen-Nürnberg, Sternwartstr 7, D-96049 Bamberg, Germany

${ }^{5}$ CSIRO Astronomy and Space Sciences, Australia Telescope National Facility, PO Box 76, Epping, NSW 1710, Australia

${ }^{6}$ Department of Astronomy, Faculty of Mathematics, University of Belgrade, Studentski trg 16, 11000 Belgrade, Serbia

${ }^{7}$ School of Cosmic Physics, Dublin Institute for Advanced Studies, 31 Fitzwillam Place, Dublin 2, Ireland

${ }^{8}$ School of Science, University of New South Wales, Australian Defence Force Academy, Canberra, ACT 2600, Australia

${ }^{9}$ Isaac Newton Institute of Chile, Yugoslavia Branch 
${ }^{10}$ School of Physical Sciences, The University of Adelaide, Adelaide, SA 5005, Australia

${ }^{11}$ International Centre for Radio Astronomy Research, University of Western Australia, 35 Stirling Hwy, Crawley, WA 6009, Australia

${ }^{12}$ ARC Centre of Excellence for All Sky Astrophysics in 3 Dimensions (ASTRO 3D), Australia

${ }^{13}$ International Centre for Radio Astronomy Research, Curtin University, Bentley, WA 6102, Australia

${ }^{14}$ Astrophysics, Cosmology and Gravity Centre (ACGC), Astronomy Department, University of Cape Town, Private Bag X3, Rondebosch 7701, South Africa

${ }^{15}$ Dominion Radio Astrophysical Observatory, Herzberg Programs in Astronomy and Astrophysics, National Research Council Canada, PO Box 248, Penticton, BC V2A 6J9, Canada
${ }^{16}$ Institute for Advanced Research, Nagoya University, Chikusa-ku, Nagoya 464-8601, Japan

${ }^{17}$ Space Science Center, Department of Earth and Space Sciences, Morehead State University, Morehead, KY 40351, USA

${ }^{18}$ ASTRON - Netherlands Institute for Radio Astronomy, NL-7991 PD Dwingeloo, the Netherlands

${ }^{19}$ Research School of Astronomy \& Astrophysics, Australian National University, Canberra, ACT 2610, Australia

${ }^{20}$ Instituto de Astronomía Teórica y Experimental - Observatorio Astronómico Có́doba (IATE-OAC-UNC-CONICET), Laprida 854, X5000BGR Córdoba, Argentina

This paper has been typeset from a $\mathrm{T}_{\mathrm{E}} \mathrm{X} / \mathrm{L} \mathrm{T}_{\mathrm{E}} \mathrm{X}$ file prepared by the author. 